\title{
Interferon-Dependent Induction of Clr-b during Mouse Cytomegalovirus Infection Protects Bystander Cells from Natural Killer Cells via NKR-P1B-Mediated Inhibition
}

\author{
Christina L. Kirkham ${ }^{a}$ Oscar A. Aguilar ${ }^{a}$ Tao Yu ${ }^{b}$ Miho Tanaka ${ }^{a} \quad$ Aruz Mesci $^{a}$ \\ Kuan-Lun Chu ${ }^{a}$ Jason H. Fine ${ }^{a}$ Karen L. Mossman ${ }^{c}$ Rod Bremner ${ }^{b}$ \\ David S.J. Allan ${ }^{a}$ James R. Carlyle ${ }^{a}$ \\ a Department of Immunology, University of Toronto, and Sunnybrook Research Institute, Toronto, ON, and \\ bLunenfeld Tanenbaum Research Institute, Sinai Health System, Department of Laboratory Medicine and \\ Pathobiology, and Department of Ophthalmology and Vision Sciences, University of Toronto, Toronto, ON, and \\ 'McMaster Immunology Research Centre, Department of Pathology and Molecular Medicine, McMaster University, \\ Hamilton, ON, Canada
}

\section{Keywords}

Natural killer cell · Missing-self recognition - NKR-P1B · Clr-b ·

Clec2 $d \cdot$ Paracrine $\cdot$ Interferon $\cdot$ Mouse cytomegalovirus

\begin{abstract}
Natural killer (NK) cells are innate lymphocytes that aid in self-nonself discrimination by recognizing cells undergoing pathological alterations. The NKR-P1B inhibitory receptor recognizes $\mathrm{Cl} r-\mathrm{b}$, a self-encoded marker of cell health downregulated during viral infection. Here, we show that Clr-b loss during mouse cytomegalovirus (MCMV) infection is predicated by a loss of $\mathrm{Clr}-\mathrm{b}$ (Clec2d) promoter activity and nascent transcripts, driven in part by MCMV ie3 (M122) activity. In contrast, uninfected bystander cells near MCMV-infected fibroblasts reciprocally upregulate Clr-b expression due to paracrine type-I interferon (IFN) signaling. Exposure of fibroblasts to type-I IFN augments Clec2d promoter activity and nascent Clr-b transcripts, dependent upon a cluster of IRF3/7/9 motifs located $\sim 200$ bp upstream of the tran-
\end{abstract}

\section{KARGER}

(C) 2017 S. Karger AG, Basel

E-Mail karger@karger.com

www.karger.com/jin scriptional start site. Cells deficient in type-I IFN signaling components revealed IRF9 and STAT1 as key transcription factors involved in Clr-b upregulation. In chromatin immunoprecipitation experiments, the Clec $2 d$ IRF cluster recruited STAT2 upon IFN-a exposure, confirming the involvement of ISGF3 (IRF9/STAT1/STAT2) in positively regulating the Clec2d promoter. These findings demonstrate that Clr-b is an IFNstimulated gene on healthy bystander cells, in addition to a missing-self marker on MCMV-infected cells, and thereby enhances the dynamic range of innate self-nonself discrimination by NK cells.

(C) 2017 S. Karger AG, Basel

\section{Introduction}

Natural killer (NK) cells are an important component of innate immune responses to pathological target cells, including tumor, virus-infected, antibody-coated, transplanted, and "stressed" cells. NK cell effector functions

Dr. James R. Carlyle

Department of Immunology, Sunnybrook Research Institute, University of Toronto 2075 Bayview Ave. (S-236)

Toronto, ON M4N 3M5 (Canada)

E-Mail james.carlyle@utoronto.ca 
include cellular cytotoxicity, mediated by exocytosis of preformed granules (containing perforin, granzymes, and granulysin), expression of apoptosis-inducing surface molecules (such as Fas-L and TRAIL), cytokine secretion [most notably interferon (IFN) $-\gamma$, TNF- $\alpha$, and GM-CSF], and chemokine responses [1]. Recently, NK cells have been reclassified as a subset of group-1 innate lymphoid cells (ILC) that share with ILC1 both T-bet (Tbx21) expression and IFN- $\gamma$ secretion yet also possess cytotoxic function and enhanced expression of eomesodermin (Eomes) [2, 3]. NK cells distinguish between healthy and pathological target cells through a complex integration of signaling events mediated by inhibitory and stimulatory cell surface receptors, which in turn recognize cognate ligands either downregulated or induced on target cells under surveillance [1].

In mice, self-specific NK cell receptors (NKR) include the Ly49 (Klra) family of inhibitory and activating receptors that mainly recognize classical MHC-I molecules, the bifunctional CD94/NKG2 (Klrd1/Klrc) family that recognizes nonclassical MHC-I molecules, the stimulatory NKG2D (Klrk1) receptor that recognizes MHC-I-related stress ligands, and the NKR-P1 (Klrb) family of inhibitory and stimulatory receptors that recognize C-type-lectin-related (Clr/Clec2) glycoproteins [4]. All of these receptors are encoded within the NK gene complex (NKC) located on mouse chromosome 6 , rat chromosome 4, and human chromosome 12 [5], yet a number of loci encoding other NKR, including NKp46 (Ncr1) and 2B4 (Cd244), are located outside the NKC [reviewed in 6].

Among this diversity, the NKR-P1 receptor family is somewhat conspicuous in being genetically linked to its cognate Clr ligand family, akin to one other MHC-independent recognition system, i.e., CD244:CD48; this arrangement may ensure co-inheritance of self-specific receptor-ligand interactions [7-9]. In the mouse, 5 functional NKR-P1 receptors have been identified, including the activating receptors NKR-P1A (Klrbla; unknown ligand), NKR-P1C (NK1.1; Klrb1c; unknown ligand), and NKR-P1F (Klrb1f; recognizes Clr-c,d,g), as well as the inhibitory isoforms NKR-P1B/D (Klrblb; recognizes Clr-b) and NKR-P1G (Klrb1g; recognizes Clr-d,f,g) [10]. At least one other receptor pseudogene locus is annotated (NKRP1E; Klrb1-ps1), while other Clr loci with unknown or pseudogene function also exist (Clr-a,e,h,i,j) [11, 12]. In humans, only a single inhibitory NKR-P1A receptor exists (CD161/KLRB1), genetically linked to its cognate ligand (LLT1/CLEC2D); however, related stimulatory receptors are also encoded within the NKC, tightly linked to their respective ligand loci, including NKp80 (KLRF1; ligand, AICL/CLEC2B) and NKp65 (KLRF2; ligand, KACL/CLEC2A) [13].

The inhibitory NKR-P1B:Clr-b interaction is currently the most well-characterized recognition pair. Like the MHC-I-specific Ly49 receptors, NKR-P1B:Clr-b interactions are involved in both NK cell education and target cell recognition $[14,15]$. Clr-b $(\mathrm{Clec} 2 d)$ is a type-II transmembrane C-type lectin-like glycoprotein expressed on most hematopoietic and some nonhematopoietic cells in a pattern similar to that of MHC-I molecules [8]. However, under pathological conditions such as oncogenesis, virus infection, and genotoxic stress, Clr-b is rapidly downregulated at both the cell surface and steady-state transcript levels, rendering these cells more sensitive to NK cell-mediated "missing-self" recognition [8, 16-19]. The regulatory mechanisms governing the modulation of Clr-b expression remain to be elucidated, although previous work has suggested roles for transcriptional and posttranscriptional, as well as ubiquitin-proteasomal and endolysosomal, control [16-19].

To investigate the genomic control of the Clr-b/Clec $2 d$ gene at the promoter and nascent transcript levels in healthy versus virus-infected cells, we used MCMV as a model pathogen. MCMV is a $\beta$-herpesvirus with a large double-stranded DNA genome capable of accommodating numerous immunoevasin genes that subvert host immune responses. Previous studies have shown that MCMV, RCMV-E, and vaccinia virus infections promote a rapid loss of mouse $\mathrm{Clr}-\mathrm{b} / \mathrm{Clec} 2 \mathrm{~d}$ and rat Clr-11/Clec2d 11 on fibroblasts $[16,18,19]$. Interestingly, at early time points during MCMV infection in vitro, uninfected fibroblasts actually upregulate Clr-b expression, as do cells exposed to passaged viral supernatants. This reciprocal regulation may represent a means to ensure optimal selfnonself discrimination between uninfected "bystander" cells in the vicinity of infected "missing-self" targets.

Here, we demonstrate that MCMV-mediated downregulation of Clr-b steady-state transcripts is controlled by disruption of Clec $2 d$ promoter activity, mediated at least in part by the cell-autonomous action of the MCMV $i e 3$ gene product in trans. In contrast, Clr-b upregulation on uninfected bystander cells is driven by paracrine typeI interferon (IFN $\alpha \beta$ ) in a manner that is dependent upon IFNAR1 signaling and occupancy of the Clec $2 d$ promoter in cis by a complex containing IRF9, STAT1, and STAT2, most likely the ISGF3 heterotrimer (IRF9/STAT1/ STAT2). Discerning how NKR ligands are regulated on both healthy and pathological target cells is an important facet in further understanding NK recognition and harnessing NK cell activity in disease therapy. 


\section{Materials and Methods}

\section{Animals}

Stat ${ }^{-1-}$ and wild-type (WT) control mice (129S6 strain) were purchased from Taconic Biosciences and handled in accordance with approved animal care protocols at Sunnybrook Research Institute.

\section{Cell Culture and Reagents}

NIH3T3 cells were purchased from the American Type Culture Collection and maintained in DMEM (HyClone) with 10\% FCS (Gibco) supplemented with $10 \mathrm{~mm}$ HEPES (HyClone), $1 \mathrm{~mm}$ sodium pyruvate (HyClone), $50 \mu \mathrm{g} / \mathrm{mL}$ gentamicin sulfate (GIBCO), 100 $\mathrm{U} / \mathrm{mL}$ penicillin (HyClone), $100 \mu \mathrm{g} / \mathrm{mL}$ streptomycin (HyClone), 2 mM GlutaMAX (Gibco), and $50 \mu \mathrm{M}$ 2-mercaptoethanol (Gibco). To generate stable NIH3T3 cell lines, cells were electroporated with Amaxa Nucleofector II (see below) with linearized plasmid and selected using $2.5 \mu \mathrm{g} / \mathrm{mL}$ puromycin (Life Technologies). Puromycin was removed for $24 \mathrm{~h}$ prior to the experiments. Primary mouse embryonic fibroblasts (MEF) were generated in the lab of Dr. Karen Mossman (McMaster University) and grown in aMEM with $15 \%$ FCS and supplemented with $1 \mathrm{~mm}$ sodium pyruvate (HyClone) and 2 mM GlutaMAX (Gibco). MEF cells were validated for knockout status by PCR following Jackson Laboratory protocols. Primary splenocytes and bone marrow cells were harvested from Stat $1^{-/-}$and WT mice, red blood cells were lysed using ACK lysis buffer, and cells

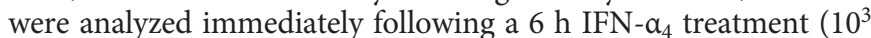
units $/ \mathrm{mL}$ ). Adult ear fibroblasts (AEF) were generated from minced ear tissue, dissociated, and cultured in 10\% supplemented DMEM. Primary AEF cells were treated with or without the STAT1 inhibitors, nifuroxazide or fludarabine (Cedarlane Labs), at titrated doses ( $50 \mu \mathrm{M}$ is shown) during IFN- $\alpha_{4}$ treatment. All cells were maintained in a subconfluent state and grown at $37^{\circ} \mathrm{C}$ and $5 \% \mathrm{CO}_{2}$.

Murine IFN- $\alpha_{4}$ was provided by Dr. Eleanor N. Fish (University of Toronto). MCMV-Smith and MCMV-GFP were described previously $[20,21]$ and in vitro passaged in MEF cells in our laboratory. For most MCMV infections or IFN- $\alpha_{4}$ treatments, $2 \times 10^{5}$ cells were plated in $1 \mathrm{~mL}$ medium and infected with MCMV (multiplicity of infection $\sim 0.5 \mathrm{PFU} /$ cell, spin-fected by centrifugation at $800 \mathrm{~g}$ for $30 \mathrm{~min}$ at $\left.37^{\circ} \mathrm{C}\right)$ or exposed to IFN- $\alpha_{4}\left(10^{3} \mathrm{U} / \mathrm{mL}\right)$ for $24 \mathrm{~h}$, unless otherwise indicated.

A piggyBac tetracycline-inducible system [22] was modified to replace the $\beta$-geo cassette with a puromycin resistance gene $\left(\right.$ Puro $\left.^{\mathrm{R}}\right)$; this vector was then used to generate doxycycline (Dox)-inducible NIH3T3 stable transfectants. Briefly, NIH3T3 cells were transiently transfected with the modified PB-TET vector containing viral ORF of interest, plus $\mathrm{PB}$ transposase and reverse transactivator (rtTA) vectors at a 1:1:1 ratio. Dox was added at a concentration of $1.5 \mu \mathrm{g} /$ $\mathrm{mL}$ the next day, and then the cells were selected in $2.5 \mu \mathrm{g} / \mathrm{mL} \mathrm{pu}$ romycin plus $1.5 \mu \mathrm{g} / \mathrm{mL}$ Dox for 5 days and allowed to recover for 2 days in $10 \%$ dMEM before being used in the experiments.

\section{Flow Cytometry}

Surface Clr-b was detected using biotinylated Clr-b mAb (4A6) [8], and IFNAR1 was detected using biotinylated mAb (MAR15A3) (BioLegend) plus secondary streptavidin-allophycocyanin (Life Technologies). Cells were stained, washed, and analyzed [23] using BD FACSCalibur and FlowJo software (TreeStar). All flow plots show cells gated by forward scatter, side scatter, lack of propidium iodide uptake, and GFP expression, where necessary.
Vector Construction

Respective B6-strain Clec2d upstream regulatory regions were cloned from BAC RP24-384I3 (BacPac Resources) using specific primers (online suppl. Table 1; for all online suppl. material, see www.karger.com/doi/10.1159/000454926) and AccuPrime HiFi Taq (Life Technologies). The mutated 500-bp promoter PCR product was generated by GeneSOE using the indicated primers (online suppl. Table 1) and Expand ${ }^{\text {PLUS }}$ High-Fidelity enzyme (Roche). The mutated sequence was validated by 2 independent transcription factor search algorithms to be devoid of transcription factor binding sites [24] (http://diyhpl.us/ bryan/irc/protocol-online/protocol-cache/TFSEARCH.html). PCR products were cloned into the luciferase vectors pGL3-Basic or pGL4.22 (Promega) and then sequenced (Macrogen Inc., South Korea, or TCAG Sequencing, Hospital for Sick Children, Toronto, ON, Canada). The pGL4.22 reporter vector was modified to contain a puromycin resistance cassette. The $\mathrm{pRL}-\mathrm{TK}$ vector was used as a control for transfection efficiency (Promega).

To overexpress IRF3/7/9, their respective coding sequences were PCR amplified from MCMV-infected NIH3T3 cDNA using the Q5 enzyme (New England Biolabs) and the primers listed in online supplementary Table 1 . The sequences were ligated into pcDNA3.1 (Life Technologies) and sequenced. M27 and the immediate early genes were amplified as described above from MCMV-infected NIH3T3 cells and ligated into pIRES2-GFP for transient transfectants and the modified piggyBac tetracycline-inducible system [22] for stable transfectants.

\section{DNA Transfections}

NIH3T3 cells were electroporated using program U-030 on a Nucleofector II machine with the Nucleofector ${ }^{\circledR}$ Kit R (Lonza) according to the manufacturer's protocol. MEF cells were electroporated using a 10- $\mu \mathrm{L}$ Neon Transfection Kit (Life Technologies) with buffer $\mathrm{R}$ and the following program: $1,350 \mathrm{~V}, 30 \mathrm{~ms}$, and 1 pulse. The cells received a total of $1 \mu \mathrm{g}$ DNA with a molar ratio of 10:1 (pGL3:pRL-TK) for transient luciferase reporter assays. BWZ.36 reporter cells were transfected with the $10-\mu \mathrm{L}$ Neon Transfection Kit with buffer $\mathrm{R}$ and the following program: 1,400 $\mathrm{V}, 20 \mathrm{~ms}$, and 2 pulses.

\section{Intronic $q R T-P C R$}

Total RNA was extracted from NIH3T3 cells using an RNeasy Plus Mini Kit (Qiagen) following the manufacturer's instructions. The RNA was digested with DNaseI (Life Technologies) and reverse transcribed using a Super-Script III Kit, with random hexamer primers (Life Technologies). qRT-PCR was performed using SsoFast EvaGreen Supermix (Bio-Rad) or PerfeCTa SYBR Green Supermix (Quanta BioSciences) on a CFX96 (Bio-Rad) instrument. For SsoFast EvaGreen, the cycling conditions were $95^{\circ} \mathrm{C}$ for $30 \mathrm{~s}$ and $40 \mathrm{cycles}$ of $95^{\circ} \mathrm{C}$ for $5 \mathrm{~s}$ and $60^{\circ} \mathrm{C}$ for $5 \mathrm{~s}$. For PerfecTa, the cycling conditions were $95^{\circ} \mathrm{C}$ for $3 \mathrm{~min}$ and 40 cycles of $95^{\circ} \mathrm{C}$ for $10 \mathrm{~s}$ and $60^{\circ} \mathrm{C}$ for $30 \mathrm{~s}$. PCR products were confirmed by melting curve analysis. The primers used for intronic qRT-PCR are listed in online supplementary Table 1 . For all qRT-PCR experiments, primers targeting 5 independent housekeeping genes were tested for each condition, with the least variable gene(s) being selected as reference gene(s) for those conditions. Analysis was done using CFX Manager software (Bio-Rad) and validated by manual calculations. 
Luciferase Bioluminescence and Protein Assays

Following transfection, NIH3T3 or MEF cells were plated in 6-well plates or 24-well plates, respectively. After overnight incubation, cells were treated with IFN- $\alpha_{4}$ or infected with MCMV for $24 \mathrm{~h}$. Transiently transfected cells were assayed using the Dual Luciferase reporter system (Promega) and a Varioskan microplate reader (Thermo Scientific). Transient transfection pGL3 vector results represent the averaged ratios of firefly (Photinus) to Renilla (sea pansy) relative light units (RLU), subsequently normalized to values obtained using the pGL3 empty vector, where indicated. Stably transfected cells were assayed using the Luciferase Reporter Assay System (Promega) and results represent the average firefly RLU values normalized to total protein per sample and indexed relative to empty vector values, where indicated.

\section{Chromatin Immunoprecipitation}

NIH3T3 cells $\left(10 \times 10^{6}\right)$ were treated with IFN- $\alpha_{4}\left(10^{3} \mathrm{U} / \mathrm{mL}\right)$ or left untreated for $1.5 \mathrm{~h}$. ChIP was performed as described previously [25]. Briefly, cells were cross-linked with $1 \%$ formaldehyde at room temperature for $10 \mathrm{~min}$, washed twice with ice-cold PBS, collected in $1 \mathrm{~mL}$ PBS, and spun down. Cells were resuspended in $1 \mathrm{~mL}$ lysis buffer [1\% SDS, $10 \mathrm{~mm}$ EDTA, and $50 \mathrm{~mm}$ Tris- $\mathrm{HCl}(\mathrm{pH}$ 8)] plus proteinase inhibitors, incubated on ice for $10 \mathrm{~min}$, and sonicated to an average size of $500 \mathrm{bp}$. Chromatin was precleared with $25 \mu \mathrm{L}$ Pansorbin (Merck Millipore) at $4^{\circ} \mathrm{C}$ for $15 \mathrm{~min}$. A 100$\mu \mathrm{L}$ aliquot of sonicated chromatin was immunoprecipitated with $2 \mu \mathrm{g}$ STAT2 mAb (D9J7L; Cell Signaling Technology) or normal rabbit IgG (Santa Cruz Biotechnology) at $4^{\circ} \mathrm{C}$ overnight. Immunoprecipitated samples were centrifuged at $13,200 \mathrm{rpm}$ and supernatants were incubated with $10 \mu \mathrm{L}$ Pansorbin at room temperature for $15 \mathrm{~min}$. Precipitates were washed twice sequentially for $3 \mathrm{~min}$ in $1 \times$ dialysis buffer [ $2 \mathrm{~mm}$ EDTA, $50 \mathrm{~mm}$ Tris- $\mathrm{HCl}(\mathrm{pH} 8)$, and $0.2 \%$ sarkosyl] and then in IP wash buffer 4 times [ $1 \%$ Nonidet P-40, 100 mM Tris- $\mathrm{HCl}$ (pH 9), $500 \mathrm{mM} \mathrm{LiCl} \mathrm{1 \% ,} \mathrm{and} \mathrm{deoxycholic}$ acid]. Samples were extracted twice with $150 \mu \mathrm{L}$ elution buffer $(1 \%$ SDS and $50 \mathrm{mM} \mathrm{NaHCO}$ ), heated at $65^{\circ} \mathrm{C}$ overnight to reverse cross-linking, and then DNA fragments were purified with a QIAEX II Gel Extraction Kit (Qiagen). For ChIP analysis, qPCR amplification was performed on the CFX Connect Real-Time PCR Detection System (Bio-Rad). Amplicons were detected using SYBR Green (Invitrogen) and the primers listed in online supplementary Table 1.

\section{BWZ Reporter Cell Assays}

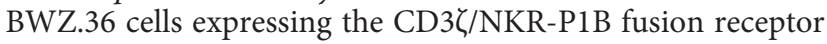
(BWZ.P1B) were used as reporter cells [8], with the following modification: the Ifnar 1 receptor gene was targeted using CRISPRCas9 technology. Oligonucleotide pairs 5' - caccg GCT GGT GGC CGG GGC GCC TT-3', or $5^{\prime}$-aaac AAG GCG CCC CGG CCA CCA GC c-3', were designed following the Zhang lab protocol (http://crispr.mit.edu) and cloned into the pSpCas9-E2A-EGFP (PX458) plasmid. Plasmids were transiently transfected into BWZ(-) or BWZ.P1B cells using the Neon Transfection System (Life Technologies). Three days after transfection, the cells were single-cell sorted on a FACSDiva for a $\mathrm{GFP}^{+}$and IFNAR1 ${ }^{-}$phenotype. Clones were subsequently validated by flow cytometry for a lack of IFNAR expression.

For reporter cell assays, NIH3T3 or MEF cells were used as stimulator cells and plated in 3-fold serial dilutions in flat-bottomed 96-well plates. Modified BWZ.P1B reporter cells (Ifnar $1^{-/-}$) were added at a density of $5 \times 10^{4}$ cells/well. Half of the co-cultures

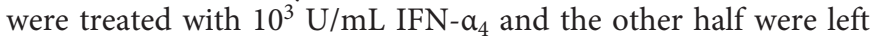
untreated. For a positive control, BWZ cells were stimulated with $10 \mathrm{ng} / \mathrm{mL}$ PMA and $0.5 \mu \mathrm{M}$ ionomycin. Purified blocking Clr-b $\mathrm{mAb}(4 \mathrm{~A} 6)$ antibody was added at $10 \mu \mathrm{g} / \mathrm{mL}$. Co-cultures were incubated overnight at $37^{\circ} \mathrm{C}$. Cells were washed with PBS and developed using $100 \mu \mathrm{L} 1 \times$ CPRG buffer [ $90 \mathrm{mg} / \mathrm{L}$ chlorophenol-red$\beta$-D-galactopyranoside (Roche), $9 \mathrm{mM} \mathrm{MgCl}_{2}, 0.1 \% \mathrm{NP}-40$, in $\mathrm{PBS}$ ] at room temperature. Optical densities (OD) were measured with a Varioskan microplate reader (Thermo Scientific) using differential $\Delta \mathrm{OD}_{595-655}$ values.

\section{Western Blots}

Proteins were extracted from NIH3T3 cells using RIPA buffer (50 mM Tris- $\mathrm{HCl}$ pH8.0, $150 \mathrm{~mm} \mathrm{NaCl}, 0.02 \%$ sodium azide, $1 \%$ NP-40, $0.1 \%$ SDS, $0.5 \%$ sodium deoxycholate) in the presence of complete protease inhibitor cocktail (Roche). Protein concentrations were quantitated using Bio-Rad protein assay reagent (BioRad). Proteins were resolved using SDS-PAGE and transferred to PVDF membranes. Membranes were preblocked with 5\% milk powder or 5\% BSA in TBS-T and blotted using STAT2 mAb (D9J7L; Cell Signaling Technology) plus secondary anti-rabbit HRP-conjugated antibody. Signals were detected using ECL (Immobilon Western HRP substrate; EMD Millipore) and a MicroChem 4.2 imager (DNR Bio-imaging Systems).

\section{Statistical Analysis}

Data were analyzed using GraphPad Prism 7 with a paired Student's $t$ test or ANOVA. All graphs show means \pm SEM. Only significant differences are annotated.

\section{Results}

\section{Infection Reciprocally Modulates Clr-b Levels} on Infected and Bystander Cells

Previous studies have shown that infection of various mouse and rat cells with a number of viruses (MCMV, RCMV-E, vaccinia, ectromelia) promotes a rapid loss of mouse Clr-b (Clec2d) and the rat Clr-b homolog rClr-11 (Clec2d11) at both the steady-state transcript and cell surface levels $[6,16,18,19]$. To distinguish between infected and uninfected (bystander) cells at the single cell level, we infected mouse NIH3T3 fibroblasts over an early time course using a modified MCMV-GFP reporter virus in which an enhanced GFP transgene is driven by an immediate early gene (ie1/3) promoter in the MCMV-Smith (VR194) strain [19-21]. While we consistently observed a loss of Clr-b surface expression on the infected population at later time points $\left(\mathrm{GFP}^{+}, 12-24 \mathrm{~h}\right.$ postinfection), at early time points the uninfected bystander population expressed elevated Clr-b surface levels relative to mock-infected parental cells (GFP- $3-12 \mathrm{~h}$ postinfection; Fig. 1a). Notably, exposure of NIH3T3 cells to UV-irradiated viral supernatants still promoted early Clr-b upregulation (data not 


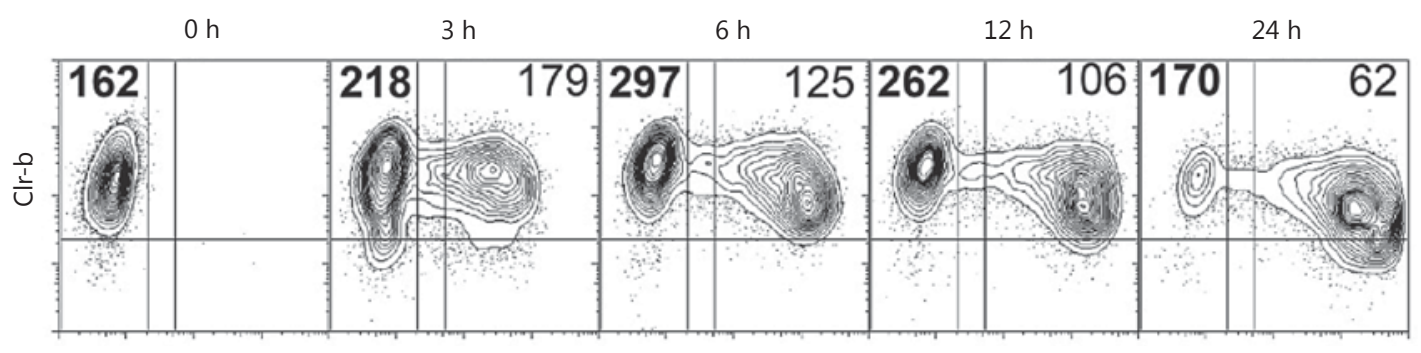

a

EGFP
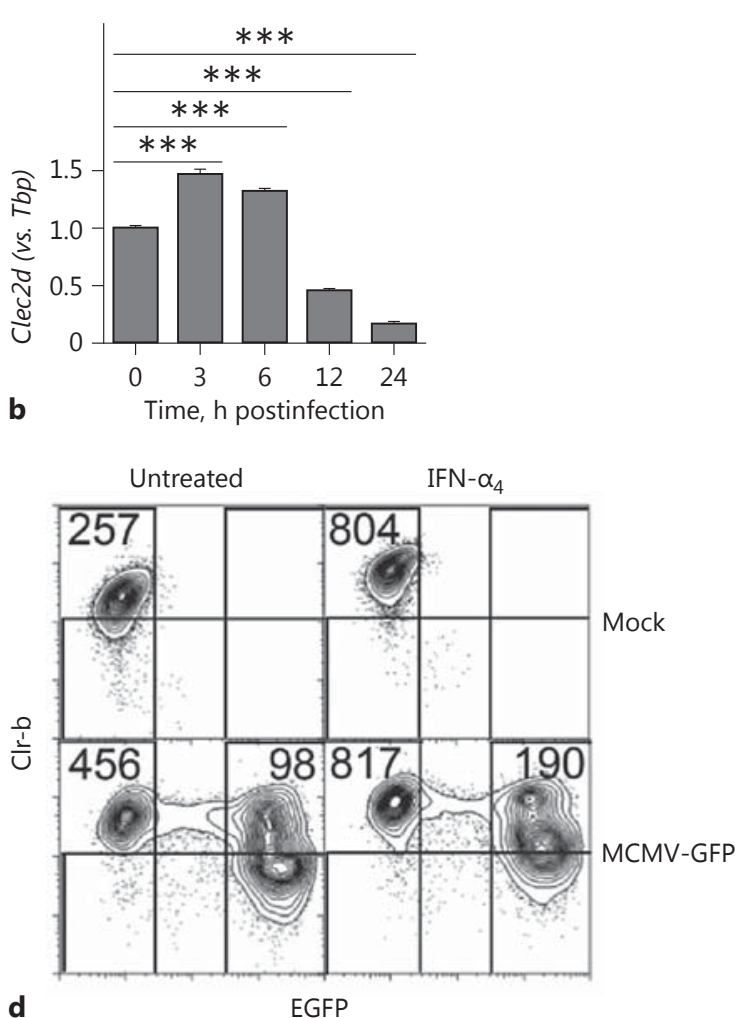

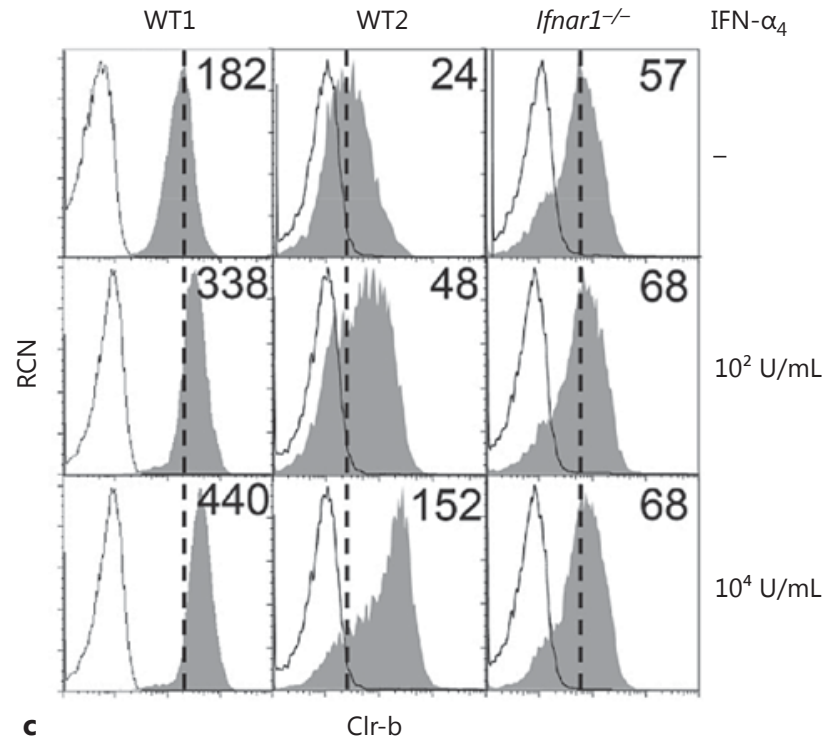

Fig. 1. Reciprocal modulation of Clr-b expression on mouse cytomegalovirus (MCMV)-infected and interferon (IFN)-stimulated bystander cells. NIH3T3 cells were infected with MCMV-GFP supernatants at a multiplicity of infection of $0.5 \mathrm{PFU} /$ cell over an extended time course. Bold numbers represent the $\mathrm{Clr}-\mathrm{b}$ median fluorescence intensity (MFI) for the uninfected cells. a Cells were analyzed by flow cytometry for Clr-b surface expression. Numbers represent median fluorescence intensities. b Quantitation of steady-state Clec $2 d$ transcripts in a by qRT-PCR normalized to Tbp expression and $0 \mathrm{~h}$. Significance was determined by a 2 -tailed $t$ test $(n=3)$. c Flow cytometric analysis of wild-type (WT) and

shown), while infection using highly purified MCMV viral particles did not cause significant bystander Clr-b upregulation [19]. This suggested that a soluble mediator in viral supernatants may promote bystander Clr-b upregulation.
Ifnar $1^{-/-}$primary mouse embryonic fibroblasts cells with or without IFN- $\alpha_{4}$ treatment for $24 \mathrm{~h}$. The shaded histogram represents Clr-b expression. Dotted lines represent the secondary reagent alone. Vertical dotted lines show median fluorescence intensities of untreated cells. Numbers represent median fluorescence intensities. d NIH3T3 cells were exposed to medium alone (untreated) or $10^{4} \mathrm{U} / \mathrm{mL}$ IFN- $\alpha_{4}$ overnight, followed by MCMV-GFP infection at a multiplicity of infection of $1 \mathrm{PFU} /$ cell or mock infection for $24 \mathrm{~h}$. The results are representative of at least 3 independent experiments. ${ }^{* * *} p<0.001$.

Time course analysis of total steady-state mRNA levels by qRT-PCR confirmed (at the population level) that MCMV-infected NIH3T3 cells initially induced and then downregulated Clr-b transcripts relative to mock-infect- 
Fig. 2. Mouse cytomegalovirus (MCMV) infection and type-I interferon (IFN) oppositely regulate $\mathrm{Clr}-\mathrm{b}$ nascent transcript levels. NIH3T3 cells were infected with MCMV-GFP at a multiplicity of infection of $0.5 \mathrm{PFU} /$ cell (a) or treated with $10^{3} \mathrm{U} /$ $\mathrm{mL}$ IFN- $\mathrm{a}_{4}$ for $24 \mathrm{~h}$ (b). Levels of Clec $2 d$ nascent pre-mRNA transcripts across introns 1-4 were quantified by qRT-PCR and normalized relative to Gapdh (a) or Hprt and $T b p(\mathbf{b})$. Significance was determined by a 2 -tailed $t$ test ( $n=3-5$ experiments). ${ }^{*} p<0.05,{ }^{* *} p<0.01,{ }^{* * *} p<0.001$.
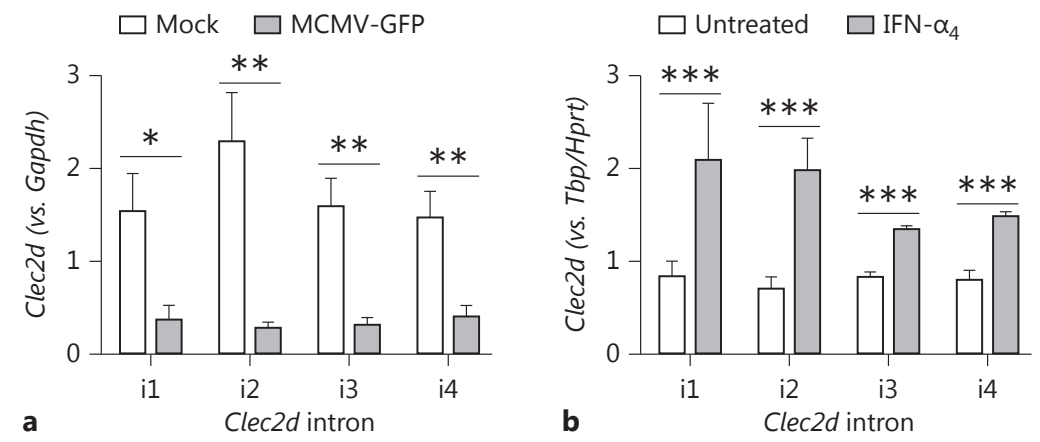

ed cells (Fig. 1b). Collectively, this suggests that MCMV infection may differentially modulate Clr-b levels in infected and bystander cells, whereby early induction of Clr-b may be due to exogenous factors produced upon MCMV infection acting in trans.

Bystander Clr-b Induction during MCMV Infection Is Type-I IFN Dependent

We hypothesized that the induced levels of Clr-b protein and Clec2d transcripts in uninfected bystander cells might be due to paracrine type-I IFN cytokine stimulation. To test this, we exposed primary murine embryonic fibroblasts (MEF) from both WT B6 and Ifnar $1^{-/-}$mice to IFN- $\alpha_{4}$ (or IFN- $\beta$; data not shown) and then examined Clr-b levels by flow cytometry. Treatment of WT MEF with IFN- $\alpha_{4}$ upregulated Clr-b ( $\sim 2$-fold at a low dose and $\sim 6$-fold at a high dose), while Ifnar $1^{-/-}$MEF showed no change in Clr-b levels (Fig. 1c). Clr-b expression also remained elevated for $\sim 2-4 \mathrm{~h}$ after IFN was removed from the culture medium (data not shown). Thus, Clr-b levels are upregulated on primary MEF cells in response to type-I IFN in an IFNAR1-dependent manner.

To test whether pre-conditioning cells with type-I IFN could block MCMV infection-mediated Clr-b loss, NIH3T3 cells were pre-treated overnight with a high dose $\left(10^{4} \mathrm{U} / \mathrm{mL}\right)$ of IFN $-\alpha_{4}$ prior to infection. Notably, IFN- $\alpha_{4}$ pre-exposure did not prevent MCMV-GFP virus infection (Fig. 1d). In addition, while an IFN-mediated induction of Clr-b was observed on all cells, infected $\left(\mathrm{GFP}^{+}\right)$ cells still displayed reduced Clr-b levels in comparison to uninfected $\left(\mathrm{GFP}^{-}\right)$cells. This suggests that the IFN-induced antiviral state is insufficient to prevent WT MCMV infection in vitro, and that infection-mediated Clr-b loss is IFN independent. To probe this reciprocal regulation further, we examined genomic control at the level of the Clr-b (Clec2d) promoter.

\section{Reciprocal Modulation of Clec2d Promoter Activity by} MCMV Infection and Type-I IFN

To elucidate the role of the Clec $2 d$ promoter, independently of mRNA stability, we performed intronic qRTPCR to assess nascent (pre-mRNA) Clec $2 d$ transcript levels, since intron removal during mRNA splicing usually occurs co-transcriptionally. Clec $2 d$ is a relatively compact gene, consisting of 5 known exons separated by 4 introns; therefore, the nascent abundance of introns 1-4 (i1-i4) was quantitated during MCMV infection relative to healthy cells. Following overnight MCMV-GFP infection, nascent Clec2d transcript levels were reduced $\sim 5$ fold relative to mock-infected cells (Fig. 2a; MCMV-GFP vs. mock). In contrast, following overnight IFN- $\alpha_{4}$ treatment, a $\sim 2$ - to 3 -fold increase in nascent Clec $2 d$ transcripts was observed (Fig. $2 b ; \mathrm{IFN}-\alpha_{4}$ vs. untreated). Thus, MCMV infection promotes a significant decrease in nascent Clr-b transcript levels, while type-I IFN treatment causes a significant increase, suggesting that $\mathrm{Clec} 2 \mathrm{~d}$ promoter activity is reciprocally modulated in infected and bystander cells.

\section{The Clec2d Promoter Is Regulated by a Functional}

Cluster of IRF Binding Sites (IRFC)

To decipher whether infected and bystander cells display differential Clec $2 d$ promoter activity, luciferase reporter assays were employed. To this end, Clec $2 d$ promoter fragments of various sizes were subcloned from a BAC vector into luciferase reporter vectors (pGL3 for transient and pGL4.22 for stable transfectants) (Fig. 3ad). NIH3T3 transfectants were subsequently exposed to 


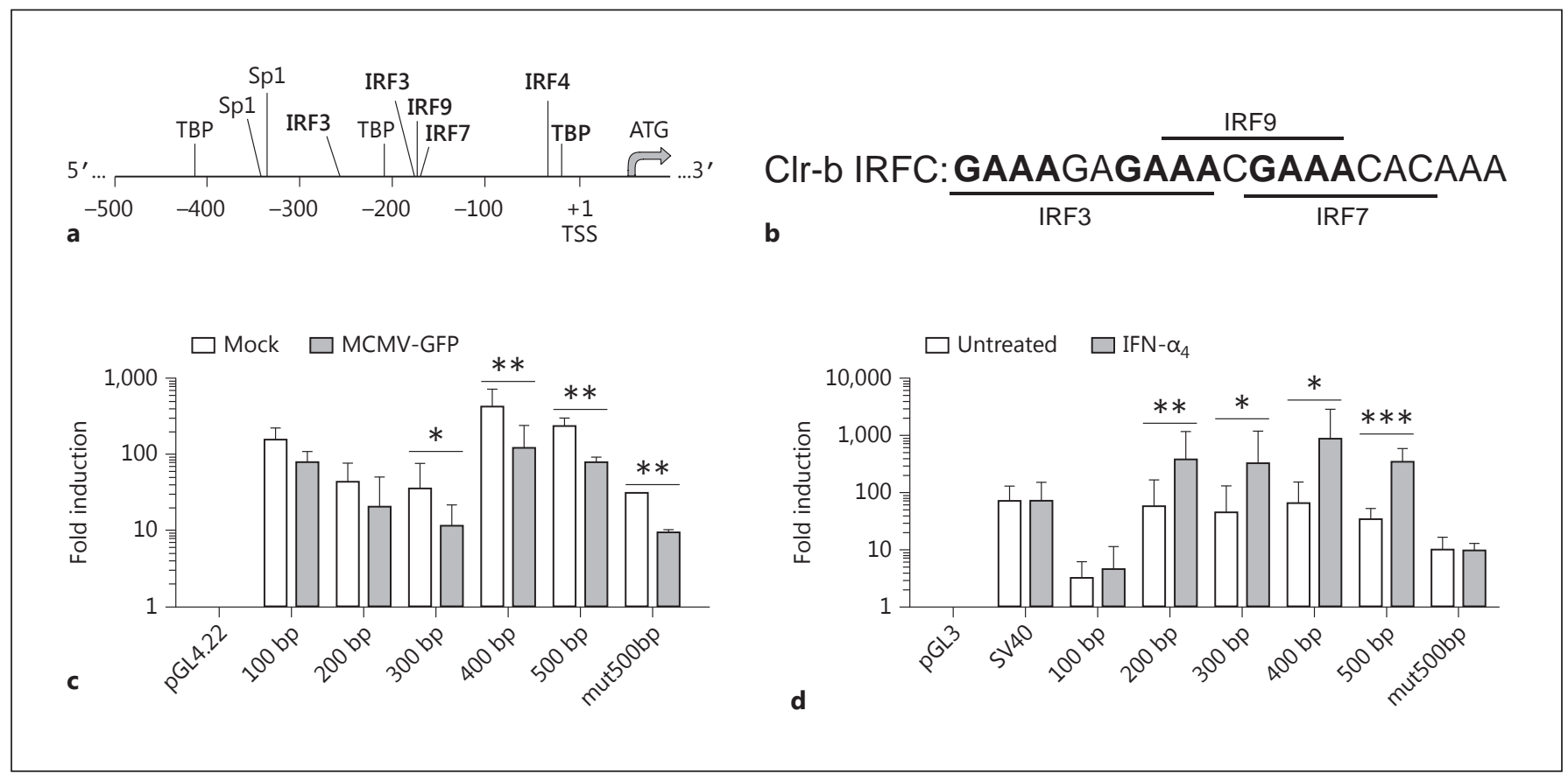

Fig. 3. Mouse cytomegalovirus (MCMV) infection and type-I interferon (IFN) reciprocally regulate Clec2d promoter activity. a Schematic representation of the Clec $2 d$ upstream regulatory region showing selected consensus motifs; ATG, translational start site; +1 , transcriptional start site (TSS); TBP, TATA binding protein; IRF, interferon regulatory factor; $S p$, specificity protein. b Nucleotide sequence of the cluster of predicted IRF-binding sites (IRF cluster) within the first $200 \mathrm{bp}$ of the Clec $2 d$ promoter. The upstream GAAA motifs in bold may enhance STAT1 (and thus ISGF3) recruitment to the IRF9 motif, in turn minimizing the requirement for the IRF3/7 motifs in response to type-I IFN [30]. c, d NIH3T3 cells were transfected with luciferase reporter plasmids containing varying sizes of the Clec $2 d$ promoter region as outlined in $\mathbf{a}$ and $\mathbf{b}$. c Stable transfectants were infected with MCMV at a multiplicity of infection of $0.5 \mathrm{PFU} /$ cell for $24 \mathrm{~h}$. d Transient transfectants were treated with $10^{3} \mathrm{U} / \mathrm{mL}$ IFN- $\alpha_{4}$ for $24 \mathrm{~h}$ the next day. Promoter activity was assayed by luciferase reporter assay relative to total protein and empty vector (pGL4.22) (b) or Renilla and empty vector (pGL3) (c). Significance was determined by a 2-tailed $t$ test on log-transformed values $(n=3-7$ experiments). ${ }^{*} p<0.05,{ }^{* *} p<0.01,{ }^{* * *} p<0.001$. either MCMV or IFN- $\alpha_{4}$ overnight and assayed for luciferase activity. As observed at the transcript level, MCMV infection caused a $\sim 4$ - to 5 -fold decrease in Clec $2 d$ promoter activity, relative to the empty pGL4.22 vector, in transfectants containing $\geq 300$ bp upstream of the Clec $2 d$ transcriptional start site (TSS) (Fig. 3c). In contrast, IFN- $\alpha_{4}$ treatment resulted in a $\sim 3$-fold increase in Clec $2 d$ promoter activity, relative to the empty pGL3 vector or the SV40 promoter, in transfectants containing $\geq 200 \mathrm{bp}$ upstream of the Clec2d TSS (Fig. 3d). Collectively, this suggests that $\mathrm{MCMV}$-mediated downregulation and IFN-mediated induction of Clec $2 d$ are regulated by distinct regulatory regions, and that DNA element(s) within the first $\sim 200$ bp upstream of the Clec $2 d$ TSS are required for IFN-mediated Clr-b induction.

We next analyzed the Clec $2 d$ promoter region to identify putative transcription factor binding sites responsible for Clr-b regulation. Several IFN regulatory factor (IRF) binding sites were predicted within the $\sim 200$ - to 300-bp promoter responsible for IFN-mediated induction (Fig. 3a, b, d). A putative IRF4 binding site was identified within the first $100 \mathrm{bp}$, although this region is not sufficient to augment Clec $2 d$ promoter activity (Fig. 3a, d). Importantly, the 200-bp promoter fragment, which is sufficient to induce $C l e c 2 d$ in response to IFN- $\alpha_{4}$, contains a cluster of overlapping binding sites for IRF3, IRF7, and IRF9 [see Fig. $3 a$ and $b$ for the IRF cluster (IRFC) nucleotide sequence]; in addition, another IRF3 binding site was predicted within the 300-bp fragment (Fig. 3a). To investigate whether the $\sim 200$-bp IRFC was necessary for typeI IFN induction, we mutated the IRF3/7/9 consensus motifs within the 500-bp promoter fragment (mut500bp) to sequences devoid of transcription factor activity (as determined by MatInspector and TfSearch). In contrast to 


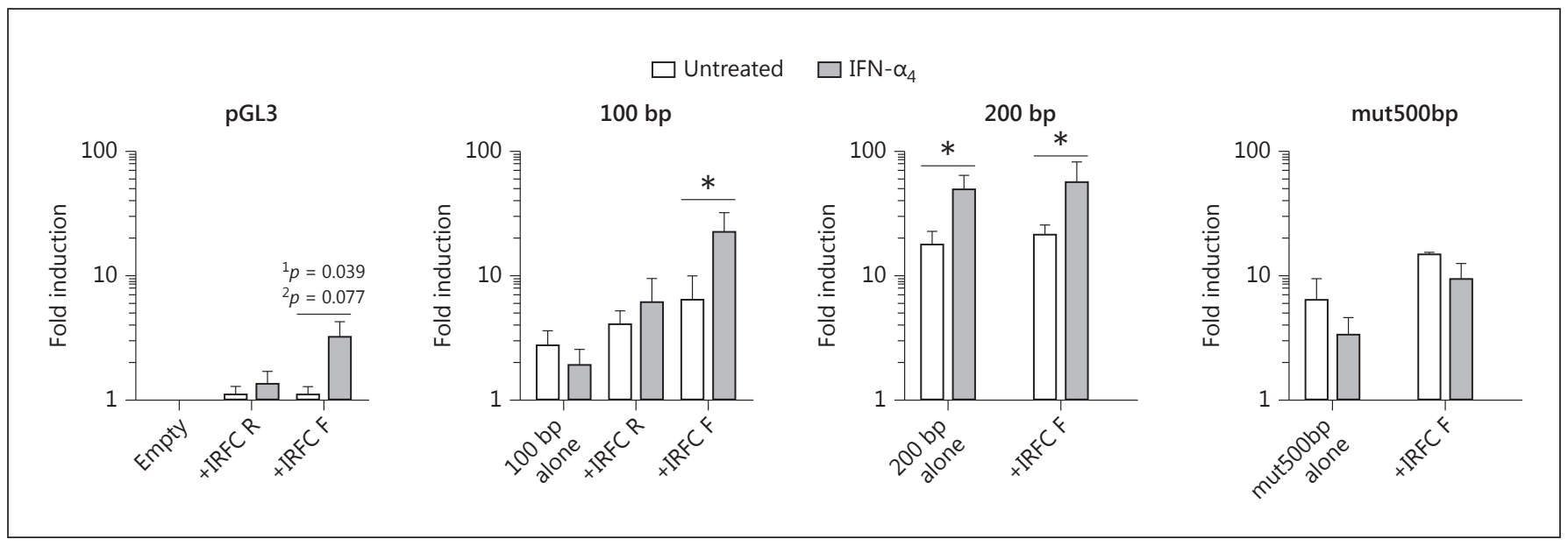

Fig. 4. An overlapping IRF3/7/9 consensus cluster (IRF cluster; IRFC) is required for type-I interferon (IFN)-dependent promoter activity. NIH3T3 cells were transfected with luciferase reporter vectors and then treated with $10^{3} \mathrm{U} / \mathrm{mL}$ IFN- $\alpha_{4}$ for $24 \mathrm{~h}$ the next day. Promoter activity was assayed by dual luciferase reporter assay relative to Renilla luciferase and normalized to the pGL3 empty vector. Significance was determined by a 1 -tailed $\left({ }^{1} p\right)$ or 2 -tailed $\left({ }^{2} p\right) t$ test on log-transformed values $\left(n=3\right.$ experiments). ${ }^{*} p<0.05$. the intact 500-bp promoter fragment, the mut500bp fragment failed to augment $\mathrm{Clec} 2 d$ promoter activity in response to IFN- $\alpha_{4}$, indicating that this region is necessary for Clr-b induction (Fig. 3d). However, reduced Clec2d promoter activity was still observed during MCMV infection using the mut500bp fragment (Fig. 3c), suggesting that virus infection more broadly affects the Clec $2 d$ promoter. Interestingly, a recent study examined whole-genome RNA polymerase II (RNAPII) occupancy by ChIPSeq in MEF cells during HSV-1 infection [26]; analysis of the Clec $2 d$ promoter from these data revealed that RNAPII was essentially absent following HSV-1 infection (online suppl. Fig. 1). Thus, the loss of Clec $2 d$ promoter activity and nascent Clr-b transcripts may be a generalized response to herpesvirus infection, and perhaps other viruses $[16,18,26]$.

Next, we examined whether the IRFC was sufficient to mediate IFN-inducible expression in a minimal promoter/enhancer assay. In transient NIH3T3 transfectants, the IRFC alone provided limited IFN-dependent induction in the forward orientation only, although this was only significant using a 1-tailed $t$ test (Fig. 4). More importantly, the IFN-nonresponsive 100-bp promoter fragment became IFN responsive upon upstream addition of a forward-oriented IRFC (Fig. 4). However, providing an additional IRFC to the IFN-responsive 200-bp promoter fragment, or adding an intact IRFC upstream of the mut$500 \mathrm{bp}$ promoter fragment, did not further increase the expression (Fig. 4). Taken together, these orientation/ proximity-dependent results suggest that the IRFC acts as part of an IFN-inducible promoter but not an independent enhancer. It remains possible that the additional IRF3 site may contribute to this context dependence.

\section{Requirement for IFNAR1, IRF9, STAT1, and STAT2}

in Clec2d Induction by Type-I IFN

To examine the mechanism of IFN-induced Clr-b induction further, we utilized MEF cells deficient in type-I IFN signaling pathway components. To this end, MEF cells from WT, Ifnar ${ }^{-/-}$, and various IRF-deficient mice were exposed to IFN- $\alpha_{4}$ or MCMV and examined for Clr$b$ levels by flow cytometry. As shown previously, IFN- $\alpha_{4}$ upregulated Clr-b on WT but not Ifnar $1^{-/-}$MEF (Fig. 5a), demonstrating a requirement for IFNAR1 signaling. IFN-dependent Clr-b induction was also observed on

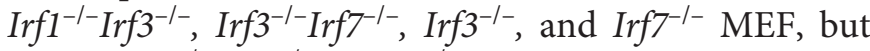
not on $\mathrm{Irf3}^{-/-} \mathrm{Irf9}^{-/-}$or Irf9 ${ }^{-/-} \mathrm{MEF}$, demonstrating a requirement for IRF9 function (Fig. 5a and data not shown).

On the other hand, upon MCMV-GFP exposure, infected $\left(\mathrm{GFP}^{+}\right) \mathrm{MEF}$ cells downregulated Clr-b independently of genotype, and WT bystander $\left(\mathrm{GFP}^{-}\right) \mathrm{MEF}$ cells routinely induced Clr-b, yet $\operatorname{Irf3^{-/}} \operatorname{IrfS}^{-/-}$and $\operatorname{Irf9^{-/-}}$ bystander $\left(\mathrm{GFP}^{-}\right) \mathrm{MEF}$ cells did not upregulate Clr-b (online suppl. Fig. 2). Results using $\operatorname{Irf3^{-/-}}$ and $\operatorname{Irf7^{-/-}} \mathrm{MEF}$ were not as clear, with bystander cells inducing Clr-b in some but not all experiments (dependent upon resting Clr-b levels and viral multiplicity of infection; online suppl. Fig. 2). Taken together, this suggests that IFN-mediat- 


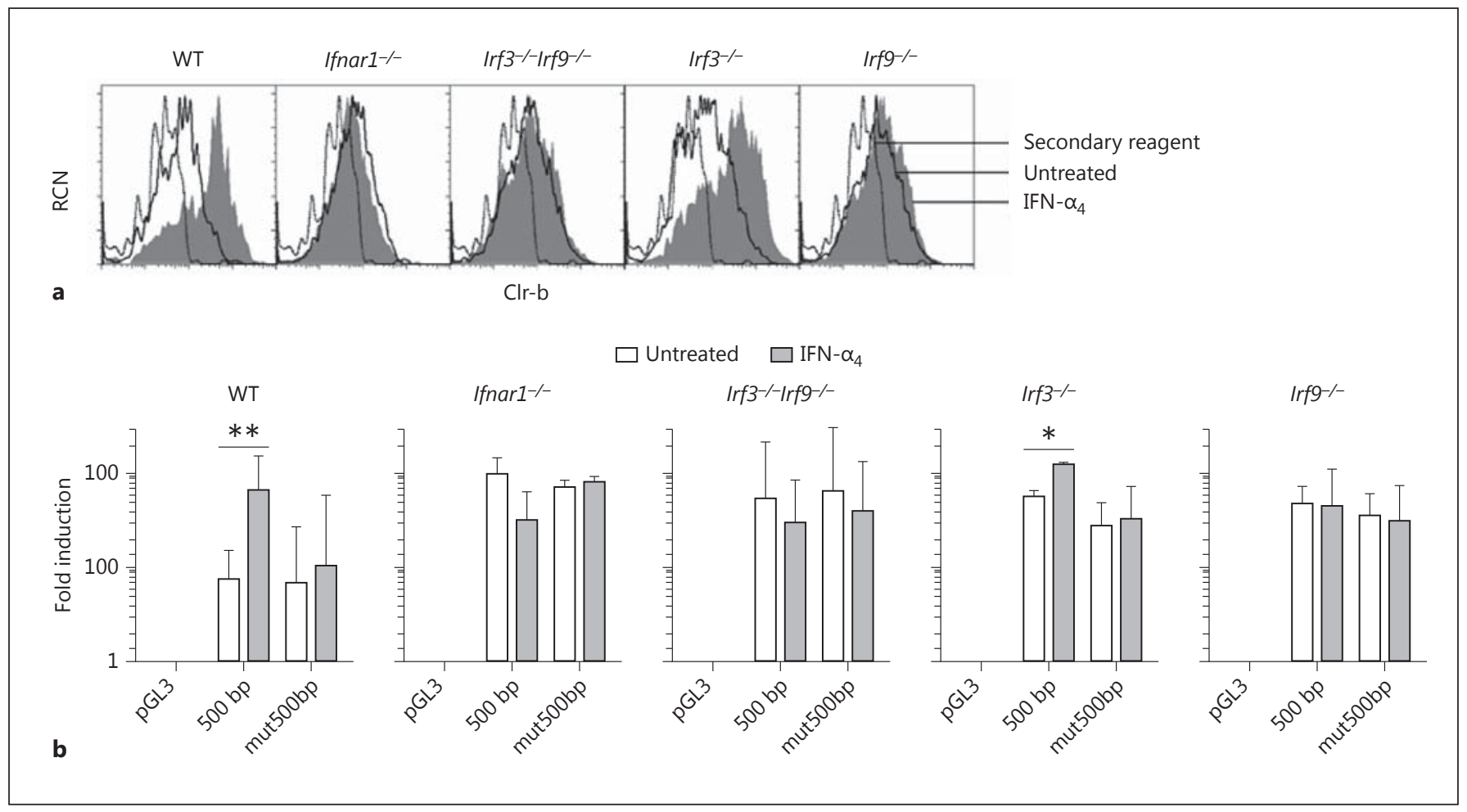

Fig. 5. Type-I interferon (IFN)-dependent induction of Clr-b is IRF9 dependent. a Primary mouse embryonic fibroblast cells derived from wild-type (WT), Ifnar1 $1^{-/-}, \operatorname{Irf3} 3^{-/} \operatorname{IrfS}^{-/-}, \operatorname{Irf3} 3^{-/-}$, and $\operatorname{Irf9^{-/-}}$ mice were treated with $10^{3} \mathrm{U} / \mathrm{mL}$ IFN- $\alpha_{4}$ for $24 \mathrm{~h}$ and analyzed for Clr-b cell surface expression by flow cytometry. The dotted line represents the secondary reagent alone; solid lines represent untreated cells; shaded histograms represent treated cells. b Mouse embryonic fibroblast cells as in a were transfected with luciferase reporter constructs and analyzed by dual luciferase reporter assay. The graphs show fold luciferase induction relative to Renilla and normalized to the pGL3 empty vector. Significance was determined by a 2 -tailed $t$ test on log-transformed values $(n=3-5$ experiments). c Mouse embryonic fibroblast cells were transfected with overexpression vectors containing IRF3, IRF7, and/or IRF9 for $24 \mathrm{~h}$ and then left untreated or

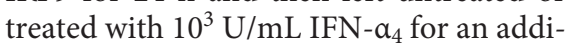
tional $24 \mathrm{~h}$ before analysis of Clr-b expression by flow cytometry. Histograms are gated on $\mathrm{GFP}^{+}$cells. Dotted lines represent the secondary reagent alone; solid lines represent untreated cells; shaded histograms represents IFN-treated cells. The results are representative of at least 4 independent experiments.

IFN-Dependent Clr-b Induction during MCMV Infection

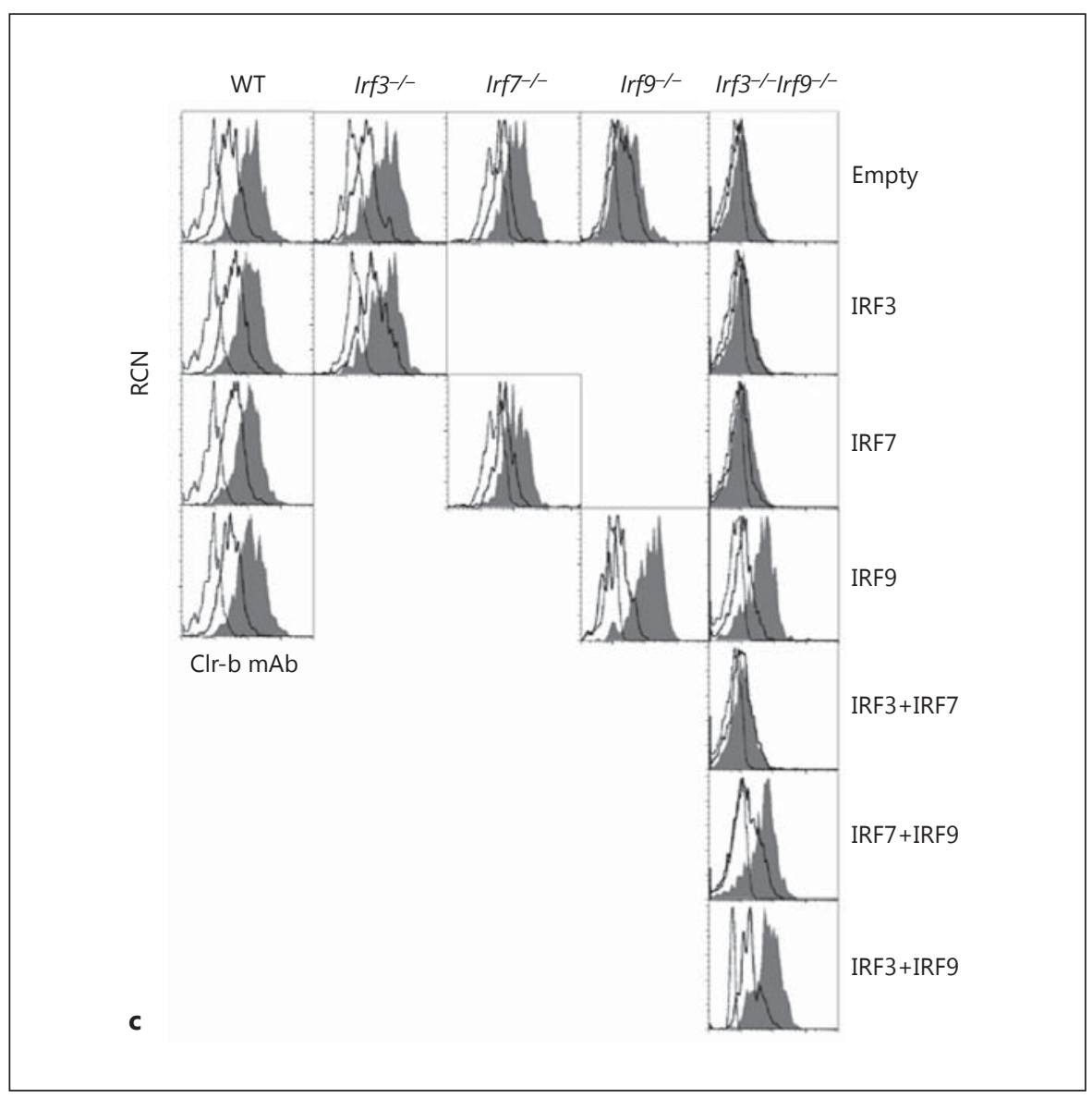

J Innate Immun 2017;9:343-358 DOI: $10.1159 / 000454926$ 
Fig. 6. Type-I interferon (IFN)-mediated Clr-b induction is dependent on STAT1. a Splenocytes (SPL), bone marrow cells (BMC), and primary adult ear fibroblasts (AEF) were harvested from wild-type (WT) and Stat ${ }^{-/-}$mice, treated with $10^{3} \mathrm{U} /$

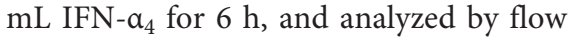
cytometry for Clr-b expression. b NIH3T3 cells were treated with vehicle alone (DMSO), $50 \mu \mathrm{M}$ nifuroxazide, or $50 \mu \mathrm{M}$ fludarabine for $22 \mathrm{~h}$, at which point $10^{3} \mathrm{U} /$ $\mathrm{mL}$ IFN- $\alpha_{4}$ was added for $3 \mathrm{~h}$ prior to flow cytometric analysis of $\mathrm{Clr}-\mathrm{b}$ expression. Dotted lines represent the secondary reagent alone; solid lines represent cells not treated with IFN- $\alpha_{4}$; shaded histograms represent IFN- $a_{4}$-treated cells. Numbers reflect the median fluorescence intensity of untreated cells (left) or IFN- $\alpha_{4}$-treated cells (right); the lower number (e.g. $8 \times$ ) represents the fold change in Clr-b MFI (IFN- $a_{4}$ treated/untreated).

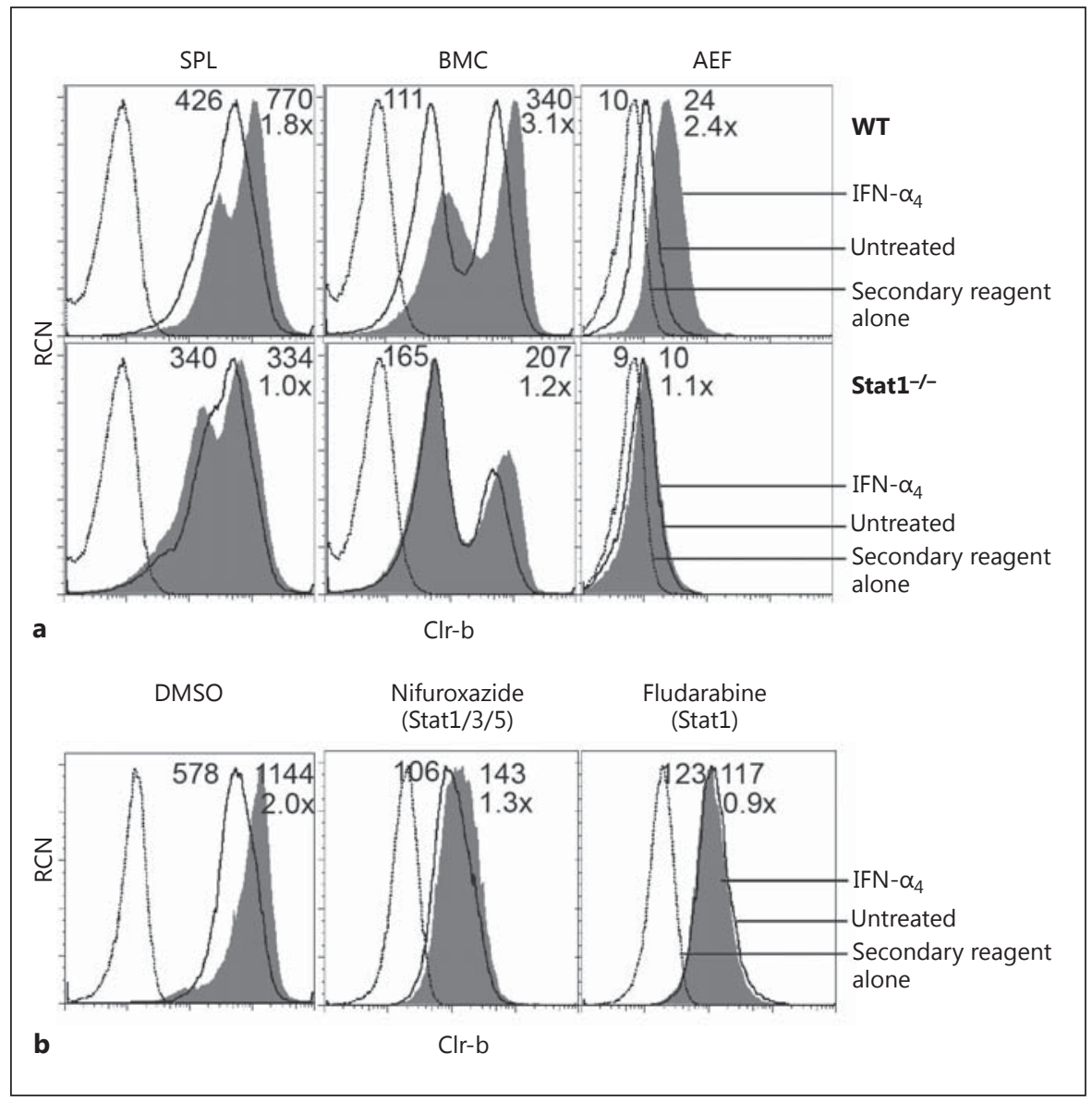

ed Clr-b induction requires IRF9, while MCMV-mediated Clr-b downregulation is more complex and appears to be IRF1/3/7/9 independent.

We next used mutant MEF cells to reanalyze Clec2d promoter activity via luciferase reporter assays. As shown previously for NIH3T3, the 500-bp Clec2d promoter fragment augmented the luciferase activity in response to IFN- $\alpha_{4}$ in WT and Irf3 ${ }^{-/-}$MEF cells, while the mut500bp fragment did not (Fig. 5b). In contrast, neither the intact 500-bp nor the mut500bp promoter fragments augmented luciferase activity in response to IFN- $\alpha_{4}$ treatment in Ifnar $1^{-/-}$, Irf3 $3^{-/}$Irf9 ${ }^{-/-}$, or Irf9 ${ }^{-/-}$MEF transfectants (Fig. 5b). This suggests that both IRF9 and the IRF3/7/9 cluster, in addition to intact IFNAR1 signaling, are required for IFNmediated Clec $2 d$ induction. Importantly, complementation studies using mutant MEF cells and overexpression of their deleted gene products demonstrated that re-intro-

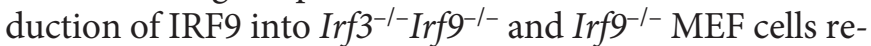
established IFN-dependent Clr-b induction, further confirming the importance of IRF9 (Fig. 5c).
IRF9 commonly activates transcription of ISG by forming a complex with STAT1 and STAT2 (termed ISGF3). To dissect this pathway further, we analyzed the IFN- $\alpha_{4}$ responses of primary cells from Stat $^{-/-}$and WT control (129S6 strain) mice. Notably, Clr-b induction on Stat $^{-1-}$ splenocytes, bone marrow cells, and primary AEF was abrogated in response to IFN- $\alpha_{4}$ treatment, while the same tissues from strain-matched (129S6) control WT mice upregulated Clr-b in response to IFN- $\alpha_{4}(1.8-, 3.1-$, and 2.4-fold, respectively; Fig. 6a). Furthermore, 2 independent STAT1 inhibitors, i.e., nifuroxazide (which inhibits STAT1/3/5 phosphorylation by Jak2/Tyk2) and fludarabine (which specifically depletes STAT1 protein and mRNA) [27-29], also blocked Clr-b upregulation upon IFN- $\alpha_{4}$ treatment of NIH3T3 cells (Fig. 6b). Taken together, these results suggest that type-I IFN-mediated Clr-b upregulation is dependent upon STAT1. Notably, it has been previously suggested that GAAA sequences localized upstream of IRF9 binding sites help to facilitate the recruitment of STAT1 and IRF9 to ISGF3-dependent 


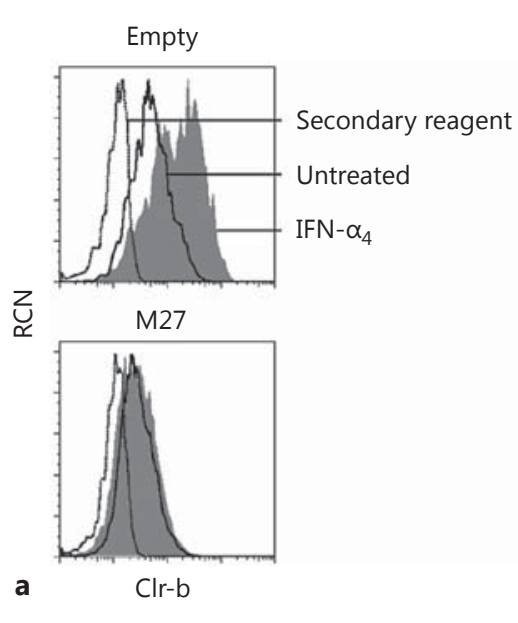

NIH3T3

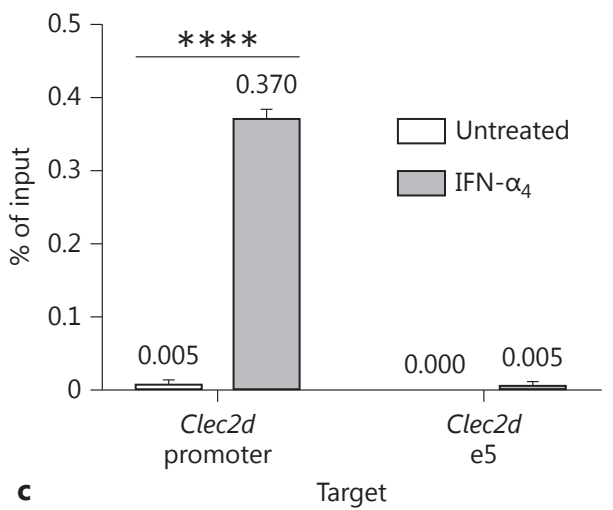

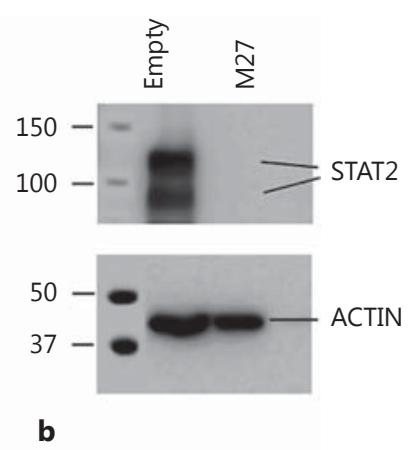

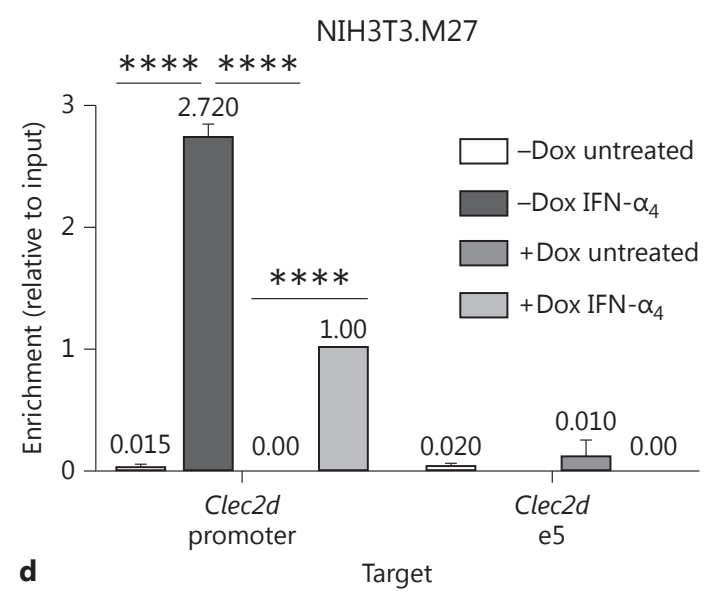

Fig. 7. STAT2 is required for Clr-b induction following type-I interferon (IFN) treatment. a NIH3T3 cells were transfected with a vector encoding the mouse cytomegalovirus M27 immunoevasin or pIRES2-GFP empty vector and then treated with $10^{3} \mathrm{U} / \mathrm{mL}$ IFN- $a_{4}$ for $24 \mathrm{~h}$ the next day. Clr-b expression was analyzed by flow cytometry, gated on $\mathrm{GFP}^{+}$cells. Dotted lines represent the secondary reagent alone; solid lines represent untreated cells; shaded histograms represent IFN-treated cells. b Cells were transfected as in a and then examined by Western blot for STAT2 expression the next day. c ChIP using an anti-STAT2 mAb was performed on NIH3T3 cells treated for $1.5 \mathrm{~h}$ with $10^{3} \mathrm{U} / \mathrm{mL}$ IFN- $\alpha_{4}$, using qRTPCR primers spanning the IRF cluster of the Clec $2 d$ promoter or exon 5 ( $n=2$ experiments). d NIH3T3 cells stably expressing M27 using a modified Dox-inducible piggyBac vector (NIH3T3.M27) were used to perform STAT2 ChIP as in c ( $n=2$ experiments). $* * * * p<0.0001$.
IFN-stimulated response elements [30]; thus, the enrichment of GAAA sequences in the IRFC (Fig. 3a, b) may explain both the STAT1 dependence and IRF3/7 independence of this cluster.

To demonstrate a role for STAT2 in type-I IFN-mediated Clec2d induction, we overexpressed the MCMV immunoevasin M27, known for its ability to target and degrade STAT2 [31], in NIH3T3 cells. Strikingly, M27 overexpression resulted in a complete loss of STAT2 protein, in turn preventing Clr-b upregulation in response to IFN treatment; thus, STAT2 is necessary for IFN-de- pendent Clr-b induction (Fig. 7a, b). To formally show recruitment of STAT2 to the Clec2d promoter, we performed chromatin immunoprecipitation (ChIP) experiments on IFN-treated NIH3T3 cells. Here, the IFN- $\alpha_{4}$ treatment was reduced to $\sim 1.5 \mathrm{~h}$, a time point reflecting an optimal increase in nascent $\mathrm{Clr}-\mathrm{b}$ transcript levels prior to protein induction (online suppl. Fig. 3a, b). Interestingly, an enrichment of Clec $2 d$ promoter gDNA (containing the IRFC) was observed upon ChIP of IFN-treated NIH3T3 cells using a STAT2 mAb (Fig. 7c), while this was not observed for downstream Clec $2 d$ exon-5 gDNA. 


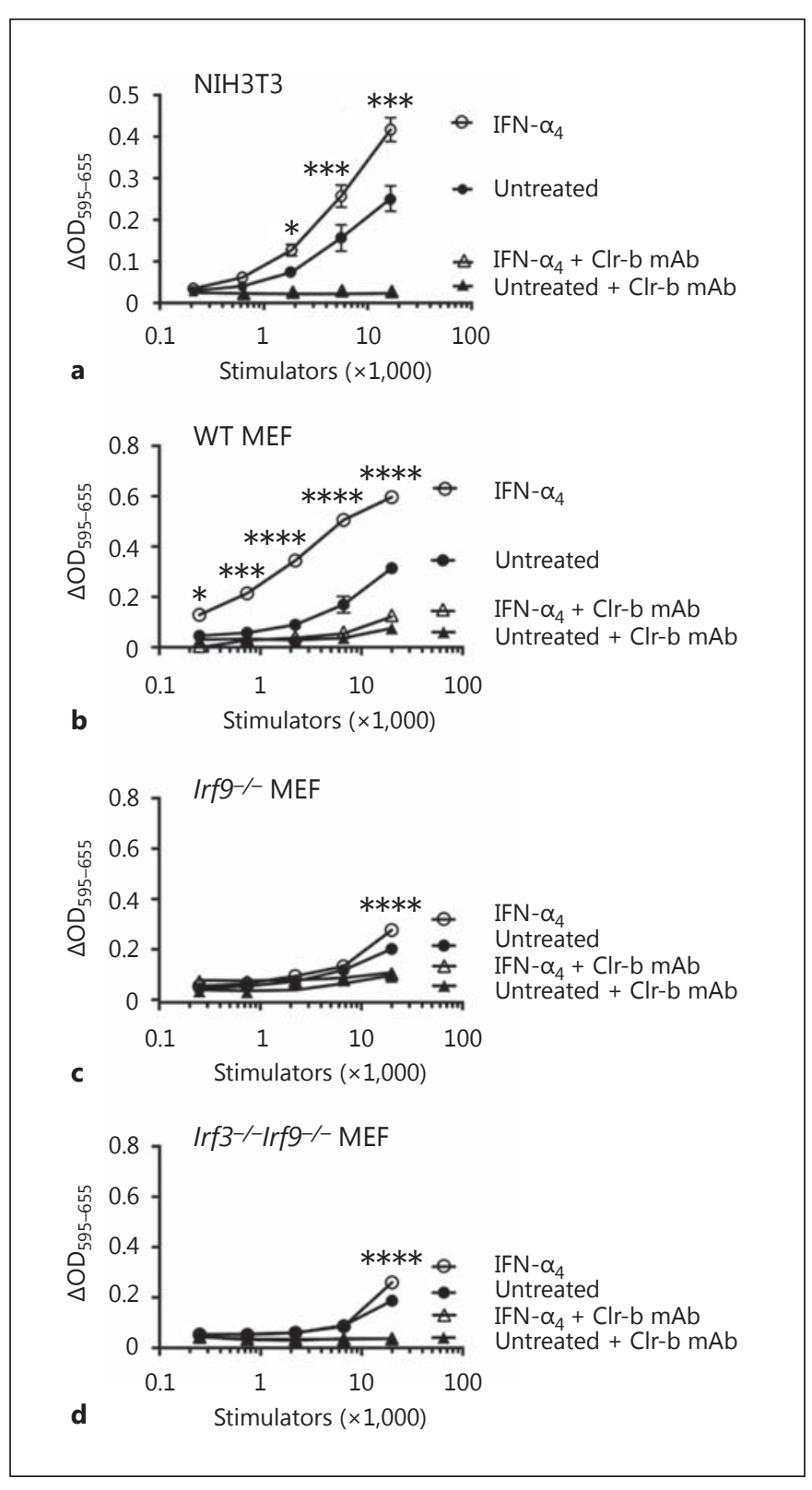

Fig. 8. BWZ.NKR-P1B reporter cell analysis of Clr-b ligand function on type-I interferon (IFN)-treated stimulator cells. NIH3T3 cells (a), primary wild-type mouse embryonic fibroblast cells (b), primary Irf $9^{-/-}$mouse embryonic fibroblast cells (c), or primary Irf3 ${ }^{-/-}$Irf $^{-/-}$mouse embryonic fibroblast cells (d) were used as stimulator cells for Ifnar1 $1^{-/} \mathrm{CD} 3 \zeta / \mathrm{NKR}-\mathrm{P} 1 \mathrm{~B}$ receptor-bearing BWZ.36 reporter cells, either untreated or treated overnight with $10^{3} \mathrm{U} / \mathrm{mL} \mathrm{IFN}-\mathrm{a}_{4}$, in the absence or presence of blocking Clr-b $\mathrm{mAb}$. Control Ifnar ${ }^{-/-}$BWZ.36 cells are not shown. Experiments were analyzed using ANOVA with Bonferroni's post hoc analysis comparing treated to untreated. The results are representative of at least 4 independent experiments, and the graphs show means \pm SEM. OD, optical density. ${ }^{*} p<0.05,{ }^{* * *} p<0.001,{ }^{* * * *} p<0.0001$.
In addition, similar results were observed using stable Dox-inducible NIH3T3.M27 transductants [22], whereby Dox induction of M27 expression mitigated the IFNdependent recruitment of STAT2 to the Clec $2 d$ promoter containing the IRFC (Fig. 7d). This demonstrates that STAT2 is directly and specifically recruited to the Clec2d promoter IRFC element upon type-I IFN treatment. Notably, attempts to perform ChIP on mouse cells using multiple anti-IRF9 antibodies and Flag-tagged IRF9 failed due to specificity and sensitivity problems (data not shown). Nonetheless, the above results collectively demonstrate that ISGF3 heterotrimer (IRF9/STAT2/ STAT1) recruitment to the IRFC is likely responsible for Clr-b induction.

\section{IFN-Dependent Clr-b Induction Augments \\ NKR-P1B-Ligand Function}

To assess the functional consequences of Clr-b upregulation by type-I IFN, we utilized BWZ reporter cell assays. Here, BWZ.36 reporter cells expressing a $\mathrm{CD} 3 \zeta$ fusion receptor of the NKR-P1B ectodomain (BWZ. P1B cells) were used to specifically quantitate NKR$\mathrm{P} 1 \mathrm{~B}$-ligand (Clr-b) function on various stimulator cells. Preliminary experiments using native BWZ.P1B cells suggested that reporter cell signaling was partially reduced by type-I IFN in a dose-dependent fashion (online suppl. Fig. $4 \mathrm{a}, \mathrm{c}$ ), possibly due to altered signaling $[32,33]$ or threshold effects on the BWZ.36 (T-lineage) cell line. Therefore, we utilized CRISPR-Cas9 gene-editing technology to generate Ifnar $^{-/-}$BWZ.P1B and control BWZ(-) cells. IFNAR1 deficiency was confirmed for multiple BWZ clones by flow cytometry using IFNAR1 mAb (online suppl. Fig. 4b). Notably, IFN- $\alpha_{4}$ treatment of NIH3T3 stimulator cells augmented NKR-P1B-ligand function using IFNAR1-deficient BWZ.P1B versus BWZ(-) cells (Fig. 8a; online suppl. Fig. 4c). This signal was Clr-b-specific, as it was blocked using Clr-b mAb.

To confirm a role for IRF9 in Clr-b induction, we repeated these assays using primary MEF from WT, $\operatorname{Irf9^{-/-}}$, and $\operatorname{Irf3} 3^{-/-} \operatorname{IrfS}^{-/-}$mice as stimulator cells. Notably, treatment of WT MEF with IFN- $\alpha_{4}$ greatly augmented BWZ.P1B reporter activity, which was blocked using Clr-b mAb (Fig. 8b). In contrast, treatment of Irf9 $9^{-{ }^{-}}$or Irf3 $3^{-/}$Irf9 $9^{-/-}$MEF with IFN- $\alpha_{4}$ yielded almost no BWZ.P1B responses, with minimal dose-dependent induction (Fig. 8c, d). Taken together, these results confirm that Irf $9^{-/-} \mathrm{MEF}$ cells possess an intrinsic functional defect in IFN-dependent induction of the NKR-P1Bligand Clr-b.
Kirkham/Aguilar/Yu/Tanaka/Mesci/Chu/ Fine/Mossman/Bremner/Allan/Carlyle 


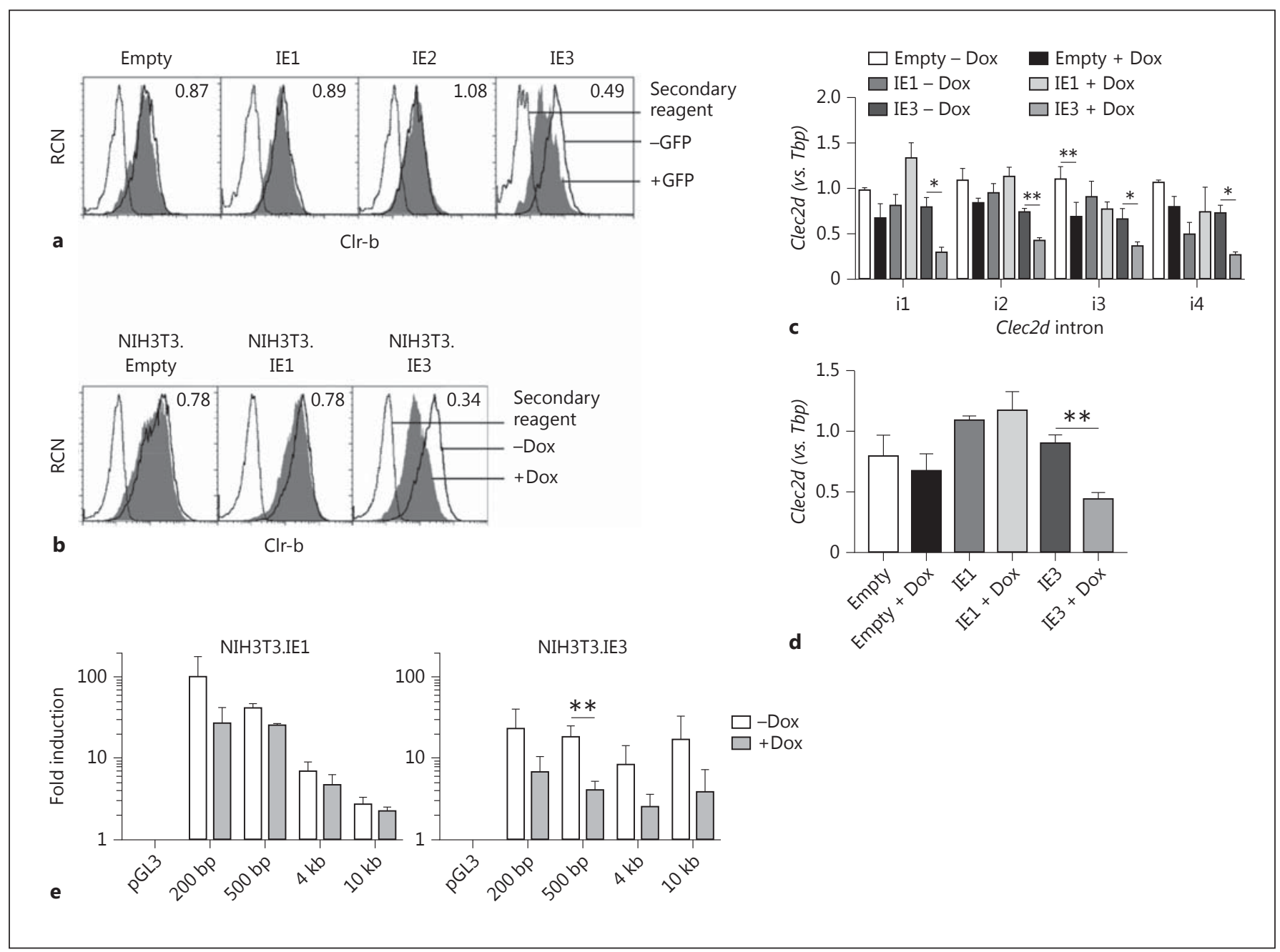

Fig. 9. Immediate early gene 3 (ie3) cell autonomously promotes downregulation of $\mathrm{Clr}-\mathrm{b}$ levels by repressing $\mathrm{Clec} 2 \mathrm{~d}$ promoter activity. a Vectors encoding the MCMV immediate early gene ORF $($ ie $1,2,3)$ or the empty pIRES2-GFP vector were transiently transfected into NIH3T3 cells and then analyzed for GFP and Clr-b expression after $24 \mathrm{~h}$. Dotted lines represent the secondary reagent alone; solid black lines represent Clr-b expression gated on $\mathrm{GFP}^{-}$ (untransfected) cells, and shaded histrograms represent Clr-b expression gated on $\mathrm{GFP}^{+}$(transfected) cells. Numbers represent the fold change in Clr-b expression $\left(\mathrm{GFP}^{+} / \mathrm{GFP}^{-}\right.$cells). $\mathbf{b}$ NIH3T3 stable transfectants of Dox-inducible piggyBac vectors encoding the ie gene products or empty vector controls were induced for 2 days in $1.5 \mu \mathrm{g} / \mathrm{mL}$ Dox and then analyzed by flow cytometry. Dotted lines represent the secondary reagent alone; solid black lines rep-

\section{The MCMV ie3 Gene Product Cell-Autonomously Represses Clec2d Expression}

In an attempt to understand mechanistically how MCMV infection downregulates Clr-b, we have tested a number of MCMV large genomic-deletion mutants and

IFN-Dependent Clr-b Induction during MCMV Infection resent Clr-b expression on untreated cells (-Dox); shaded histograms represent Clr-b expression on Dox-treated cells (+Dox). Numbers represent the fold change in Clr-b expression (+Dox/Dox). c RNA from the cells in a was analyzed by qRT-PCR for Clec $2 d$ nascent pre-mRNA transcripts of introns $1-4$ normalized relative to $\operatorname{Tb} p$ ( $n=3$ experiments). Significance was determined by a 2-tailed $t$ test. $\mathbf{d}$ RNA from the cells in $\mathbf{b}$ was analyzed for Clec $2 d$ steady-state levels by qRT-PCR relative to Tbp ( $n=3-4$ experiments). e Cells as in $\mathbf{b}$ were transfected with luciferase-reporter vectors containing various $C l e c 2 d$ promoter fragments and then promoter activity was assayed by luciferase reporter assay relative to Renilla luciferase and normalized to the empty vector control (pGL3). Significance was determined by a 2 -tailed $t$ test on logtransformed values ( $n=3$ experiments). ${ }^{*} p<0.05,{ }^{* *} p<0.01$.

cloned numerous MCMV gene products for independent overexpression. Importantly, neither $\mathrm{m} 02$ or $\mathrm{m} 145$ family immunoevasin genes nor several core genes were found to negatively regulate $\mathrm{Clr}-\mathrm{b}$ levels in isolation. However, overexpression of a single immediate-early 
gene product, ie3, but not ie 1 or $i e 2$, consistently promoted a significant $(\geq 50 \%)$ Clr-b downregulation when transiently transfected into NIH3T3 cells (Fig. 9a).

To further assess the effects of ie 3 on Clr-b levels, we generated stable Dox-inducible NIH3T3 transfectants of the alternately spliced ie1/3 genes [22]. Following Dox induction, $i e 3$ was found to promote a significant $(\geq 66 \%)$ loss of surface Clr-b, with minimal downregulation observed for ie1 and empty vector controls (Fig. 9b). To examine the effect of $i e 3$ on Clec $2 d$ gene expression, we next quantitated Clr-b nascent and steady-state transcript levels by qRT-PCR. Here, Dox-induced ie 3 expression promoted a significant decrease in both $\mathrm{Clr}-\mathrm{b}$ nascent and steady-state transcript levels ( $\geq 2$ - to 3 -fold; Fig. 9c, d). To determine whether the $i e 3$ gene product directly repressed Clec2d promoter activity, dual-luciferase constructs of the promoter fragments were transfected into the Doxinducible stable NIH3T3.IE1/3 transfectants. In line with Clr-b protein and transcript data, overexpression of $i e 3$ resulted in significantly decreased Clec $2 d$ promoter activity using the intact 500 -bp promoter construct, while ie 1 had no significant effect (Fig. 9e). Taken together, these findings suggest that the ie 3 gene product cell-autonomously represses the Clec $2 d$ promoter, in turn partially extinguishing $\mathrm{Clr}-\mathrm{b}$ nascent transcripts and downregulating surface $\mathrm{Clr}-\mathrm{b}$ protein early during MCMV infection.

\section{Discussion}

This study provides mechanistic evidence that $\mathrm{Clr}-\mathrm{b}$ $(\mathrm{Clec} 2 d)$ is an inducible IFN-stimulated gene (ISG), in addition to its demonstrated function as a "missing-self" marker of cell health that interacts with the NKR-P1B inhibitory receptor on NK cells and ILC subsets [7]. In keeping with this dual function, we propose that $\mathrm{Clr}-\mathrm{b}$ enhances self-nonself discrimination by NK cells during viral infection by two means: (i) MCMV-infected cells downregulate resting levels of Clr-b via a host pattern recognition mechanism, in order to render the "missingself" cells more susceptible to clearance via NK cell disinhibition, while (ii) normal "bystander" cells in the vicinity of infected cells induce Clr-b as a marker of cell health in response to paracrine type-I IFN, thereby reinforcing NK cell inhibition via NKR-P1B. The net effect is that NK cells encounter a broader dynamic range of Clr-b-mediated inhibition/disinhibition during self-nonself discrimination, in turn rendering them better able to integrate signaling inputs and redirect cytotoxic machinery to rec- ognize pathological versus healthy target cells in a battlefield of infection.

This work has shown that both of the above responses are facilitated at the genomic level, where significant changes in Clec2d promoter activity lead to direct and immediate changes in nascent $\mathrm{Clr}-\mathrm{b}$ transcript and protein levels. In this model, infected cells lose Clr-b in a manner partially attributable to ie3-mediated Clec2d repression, presumably involving a loss of transcription factors and/or RNAPII occupancy at the Clec2d promoter (see below). Meanwhile, infected cells secreting type-I IFN may be sensed in a paracrine manner by uninfected bystander cells via the IFNAR1 receptor. Subsequently, canonical IFNAR1 signaling activates JAK1/TYK2 to phosphorylate and activate STAT1 and STAT2 to heterotrimerize in a complex with IRF9 as ISGF3. An ISGF3-containing transcription factor complex is then recruited to the Clec2d promoter at the proximal 200-bp IRFC element (the IRF3/7/9 cluster, which acts as an IFN-stimulated response element), in turn augmenting $\mathrm{Clr}-\mathrm{b}$ nascent transcripts and protein levels. This combined regulation mechanism is significant because it creates a greater disparity between the infected and uninfected cell populations, facilitating enhanced NK cell recognition.

On the other hand, in infected cells, pattern recognition receptor (PRR) signaling events following the detection of MCMV-specific pathogen-associated molecular patterns (such as cytosolic nucleic acids), in combination with the effects of $i e 3$ on host gene transcription (and/or unidentified immunoevasins), lead directly to the loss of Clec2d promoter occupancy, Clr-b transcripts, and surface protein. While the complexity of this "missing-self" host response mechanism remains to be elucidated, the direct repressive effects of $i e 3$ on host gene expression in general and Clec $2 d$ in particular follow the tenets of a pattern recognition system, implicating the NKR-P1B:Clr-b interaction as a pattern recognition receptor axis where the pattern itself is host transcriptional integrity.

In support of this, previous studies have shown that ie3 (M122) is critical for MCMV fitness, such that without it infectious viral progeny can only be produced by cellular ie3 complementation [34-36]. Thus, MCMV (the pathogen) cannot easily mutate $i e 3$ (the molecule) to avoid cellular detection of its effects on host cells (the pattern shutting down host gene expression and turning on early viral genes) without compromising viral fitness. In turn, the Clec $2 d$ gene serves by proxy as an innate detector (the recognition axis) of the active repression of cellular host gene expression by diverse viruses. Following the shut-off
356

J Innate Immun 2017;9:343-358

DOI: $10.1159 / 000454926$
Kirkham/Aguilar/Yu/Tanaka/Mesci/Chu/ Fine/Mossman/Bremner/Allan/Carlyle 
of Clec2d, this signal is relayed to NK cells via the "missing-self" loss of Clr-b, which acts as a marker of cell health and the loss of which disinhibits (activates) NK cells via NKR-P1B (the receptor). In contrast, normal bystander cells augment $\mathrm{Clec} 2 \mathrm{~d}$ and $\mathrm{Clr}$-b expression in response to type-I IFN as a mechanism to relay to NK cells that they are indeed healthy yet responding to paracrine IFN as a byproduct of pathological infected cells in the nearby vicinity.

One remaining unanswered question is how infection with diverse viruses (CMV, HSV, and poxviruses) mechanistically promotes a rapid loss of Clec $2 d$ transcription in infected cells. One possibility is a general viral shut-off and subversion of host mRNA transcription. Another possibility is that MCMV may encode an immunoevasin that directly modulates Clr-b levels by design. Interestingly, we have previously shown that both rat CMV and MCMV encode decoy (Clr-b-surrogate) immunoevasins to elude missing-self recognition via NKR-P1B $[16,19]$, perhaps suggesting that the viruses cannot otherwise subvert general host mRNA loss and instead directly target host Clr-b and/or NKR-P1B function. Notably, while previous attempts have failed to identify CMV genes that directly lead to the loss of Clr-b expression, here we show that exogenous expression of $i e 3$ autonomously promotes Clr-b downregulation in the absence of infection. Since $i e 3$ is required for productive virus infection and early CMV gene expression [34-36], the virus cannot easily circumvent this requirement, making $\mathrm{Clr}-\mathrm{b}$ a pattern recognition axis and the NKR-P1B receptor a selfspecific pattern recognition receptor. IE3 is known to promote cell cycle arrest in $\mathrm{G}_{1} / \mathrm{G}_{0}$ and interact in MCMV replication compartments with PML, Daxx, and ND10 complex proteins involved in viral restriction, and the HCMV homolog IE2 functions to repress important host transcription factors (including HDAC and TBP/TFIID) [37-40] and it is thought to function as a transcriptionassociated factor [41]. This is interesting because the Clr$\mathrm{b}$ promoter contains an inverted TATA motif and may function as a TBP-dependent promoter. Future studies will elucidate ie3-interacting partners in host gene transcriptional regulation. It is also possible that $i e 3$ is not the sole gene involved in Clr-b downregulation during infection. Rather, since it is an immediate early gene, ie 3 likely acts as an immediate repressor of Clec $2 d$, whereas early or late gene products may then contribute to the complete loss of surface Clr-b and/or counteract the loss of Clr-b ligand or NKR-P1B inhibition specifically. Indeed, our current work implicates an m02 family member as a decoy ligand for the NKR-P1B receptor, and other ongo-

IFN-Dependent Clr-b Induction during MCMV Infection ing work implicates an m145 family member in moderately antagonizing $\mathrm{Clr}-\mathrm{b}$ downregulation during MCMV infection.

In conclusion, this work has demonstrated that Clr-b, a marker of cell health and a self ligand for the NKR-P1B inhibitory receptor, is reciprocally regulated on MCMVinfected and bystander cells. While the mechanism of "missing-self" Clr-b downregulation remains to be fully characterized, Clr-b induction on bystander cells occurs via a canonical paracrine type-I IFN signaling mechanism, making Clr-b a bona fide ISG. This dual tuning mechanism involving the inhibitory NKR-P1B:Clr-b axis facilitates NK cell self-nonself discrimination during viral infection by increasing the recognition threshold between pathological (missing-self) targets and normal (healthy-self) bystander cells.

\section{Acknowledgements}

We thank Dr. A. Makrigiannis and Dr. S. Vidal for providing us with the MCMV viruses, Dr. E. Fish and Beata Majchrzak for the IFN- $\alpha_{4}$ supernatant, and Dr. Geneve Awong, Courtney McIntosh, and Vincent Cheng for cell sorting. We also thank Drs. J.C. ZüñigaPflücker, A. Martin, A. Nagy, and T. Schrader for plasmids. C.L.K. was funded by a CGS-M Award from the Natural Sciences and Engineering Research Council of Canada (NSERC) and Ontario Graduate Scholarship (OGS) Awards. A.M. was funded by a Vanier Scholarship from the Canadian Institutes of Health Research (CIHR), and O.A.A. and J.H.F. were funded by PGS-D3 scholarships from NSERC. This work was funded by an Investigator in the Pathogenesis of Infectious Disease Award from the Burroughs Wellcome Fund and grants from the CIHR (106491 to J.R.C.).

\section{Disclosure Statement}

No competing financial interests exist.

\begin{tabular}{|c|c|}
\hline References & $\begin{array}{l}1 \text { Lanier LL: NK cell recognition. Annu Rev Im- } \\
\text { munol 2005;23:225-274. } \\
2 \text { Gordon SM, Chaix J, Rupp LJ, Wu J, Madera } \\
\text { S, Sun JC, Lindsten T, Reiner SL: The tran- } \\
\text { scription factors T-bet and Eomes control key } \\
\text { checkpoints of natural killer cell maturation. } \\
\text { Immunity 2012;36:55-67. } \\
3 \text { Spits H, Artis D, Colonna M, Diefenbach A, } \\
\text { Di Santo JP, Eberl G, Koyasu S, Locksley RM, } \\
\text { McKenzie AN, Mebius RE, Powrie F, Vivier } \\
\text { E: Innate lymphoid cells - a proposal for uni- } \\
\text { form nomenclature. Nat Rev Immunol 2013; } \\
\text { 13:145-149. } \\
4 \text { Kirkham CL, Carlyle JR: Complexity and di- } \\
\text { versity of the NKR-P1:Clr (Klrb1:Clec2) rec- } \\
\text { ognition systems. Front Immunol 2014;5:214. }\end{array}$ \\
\hline
\end{tabular}

J Innate Immun 2017;9:343-358

DOI: $10.1159 / 000454926$
357 
5 Hao L, Klein J, Nei M: Heterogeneous but conserved natural killer receptor gene complexes in four major orders of mammals. Proc Natl Acad Sci USA 2006;103:3192-3197.

6 Carrillo-Bustamante P, Kesmir C, de Boer RJ: The evolution of natural killer cell receptors. Immunogenetics 2016;68:3-18.

7 Iizuka K, Naidenko OV, Plougastel BF, Fremont DH, Yokoyama WM: Genetically linked C-type lectin-related ligands for the NKRP1 family of natural killer cell receptors. Nat Immunol 2003;4:801-807.

8 Carlyle JR, Jamieson AM, Gasser S, Clingan CS, Arase H, Raulet DH: Missing self-recognition of Ocil/Clr-b by inhibitory NKR-P1 natural killer cell receptors. Proc Natl Acad Sci USA 2004;101:3527-3532.

9 Carlyle JR, Mesci A, Fine JH, Chen P, Belanger S, Tai LH, Makrigiannis AP: Evolution of the Ly49 and Nkrp1 recognition systems. Semin Immunol 2008;20:321-330.

10 Chen P, Belanger S, Aguilar OA, Zhang Q, StLaurent A, Rahim MM, Makrigiannis AP, Carlyle JR: Analysis of the mouse 129-strain Nkrp1-Clr gene cluster reveals conservation of genomic organization and functional receptor-ligand interactions despite significant allelic polymorphism. Immunogenetics 2011; 63:627-640.

11 Plougastel B, Dubbelde C, Yokoyama WM: Cloning of Clr, a new family of lectin-like genes localized between mouse Nkrpla and Cd69. Immunogenetics 2001;53:209-214.

12 Zhang Q, Rahim MM, Allan DS, Tu MM, Belanger S, Abou-Samra E, Ma J, Sekhon HS, Fairhead T, Zein HS, Carlyle JR, Anderson SK, Makrigiannis AP: Mouse Nkrp1-Clr gene cluster sequence and expression analyses reveal conservation of tissue-specific MHC-independent immunosurveillance. PLoS One 2012;7:e50561.

13 Vogler I, Steinle A: Vis-a-vis in the NKC: genetically linked natural killer cell receptor/ligand pairs in the natural killer gene complex (NKC). J Innate Immun 2011;3:227-235.

14 Chen P, Aguilar OA, Rahim MM, Allan DS, Fine JH, Kirkham CL, Ma J, Tanaka M, Tu MM, Wight A, Kartsogiannis V, Gillespie MT, Makrigiannis AP, Carlyle JR: Genetic investigation of MHC-independent missingself recognition by mouse NK cells using an in vivo bone marrow transplantation model. J Immunol 2015;194:2909-2918.

15 Rahim MM, Chen P, Mottashed AN, Mahmoud AB, Thomas MJ, Zhu Q, Brooks CG, Kartsogiannis V, Gillespie MT, Carlyle JR, Makrigiannis AP: The mouse NKR-P1B:Clr$\mathrm{b}$ recognition system is a negative regulator of innate immune responses. Blood 2015;125: 2217-2227.

16 Voigt S, Mesci A, Ettinger J, Fine JH, Chen P, Chou W, Carlyle JR: Cytomegalovirus evasion of innate immunity by subversion of the NKR-P1B:Clr-b missing-self axis. Immunity 2007;26:617-627.

17 Fine JH, Chen P, Mesci A, Allan DS, Gasser S, Raulet DH, Carlyle JR: Chemotherapy-in- duced genotoxic stress promotes sensitivity to natural killer cell cytotoxicity by enabling missing-self recognition. Cancer Res 2010;70: 7102-7113.

18 Williams KJ, Wilson E, Davidson CL, Aguilar OA, Fu L, Carlyle JR, Burshtyn DN: Poxvirus infection-associated downregulation of Ctype lectin-related-b prevents NK cell inhibition by NK receptor protein-1B. J Immunol 2012;188:4980-4991.

19 Aguilar OA, Mesci A, Ma J, Chen P, Kirkham CL, Hundrieser J, Voigt S, Allan DS, Carlyle JR: Modulation of Clr ligand expression and NKR-P1 receptor function during murine cytomegalovirus infection. Journal of Innate Immunity 2015;7:584-600.

20 Smith MG: Propagation in tissue cultures of a cytopathogenic virus from human salivary gland virus (SGV) disease. Proc Soc Exp Biol Med 1956;92:424-430.

21 Henry SC, Schmader K, Brown TT, Miller SE, Howell DN, Daley GG, Hamilton JD: Enhanced green fluorescent protein as a marker for localizing murine cytomegalovirus in acute and latent infection. J Virol Methods 2000;89:61-73.

22 Woltjen K, Michael IP, Mohseni P, Desai R, Mileikovsky M, Hamalainen R, Cowling R, Wang W, Liu P, Gertsenstein M, Kaji K, Sung HK, Nagy A: piggyBac transposition reprograms fibroblasts to induced pluripotent stem cells. Nature 2009;458:766-770.

23 Carlyle JR, Martin A, Mehra A, Attisano L, Tsui FW, Zuniga-Pflucker JC: Mouse NKRP1B, a novel NK1.1 antigen with inhibitory function. J Immunol 1999;162:5917-5923.

24 Cartharius K, Frech K, Grote K, Klocke B, Haltmeier M, Klingenhoff A, Frisch M, Bayerlein M, Werner T: MatInspector and beyond: promoter analysis based on transcription factor binding sites. Bioinformatics 2005; 21:2933-2942.

$25 \mathrm{Ni}$ Z, Bremner R: Brahma-related gene 1-dependent STAT3 recruitment at IL-6-inducible genes. J Immunol 2007;178:345-351.

26 Abrisch RG, Eidem TM, Yakovchuk P, Kugel JF, Goodrich JA: Infection by herpes simplex virus type-1 causes near-complete loss of RNA polymerase II occupancy on the host cell genome. J Virology 2015;90:2503-2513.

27 Frank DA, Mahajan S, Ritz J: Fludarabine-induced immunosuppression is associated with inhibition of STAT1 signaling. Nat Med 1999; 5:444-447.

28 Torella D, Curcio A, Gasparri C, Galuppo V, De Serio D, Surace FC, Cavaliere AL, Leone A, Coppola C, Ellison GM, Indolfi C: Fludarabine prevents smooth muscle proliferation in vitro and neointimal hyperplasia in vivo through specific inhibition of STAT-1 activation. Am J Physiol Heart Circ Physiol 2007; 292:H2935-H2943.

29 Nelson EA, Walker SR, Kepich A, Gashin LB, Hideshima T, Ikeda H, Chauhan D, Anderson KC, Frank DA: Nifuroxazide inhibits survival of multiple myeloma cells by directly inhibiting STAT3. Blood 2008;112:5095-5102.
30 Tenoever BR, Ng SL, Chua MA, McWhirter SM, Garcia-Sastre A, Maniatis T: Multiple functions of the IKK-related kinase IKKepsilon in interferon-mediated antiviral immunity. Science 2007;315:1274-1278.

31 Zimmermann A, Trilling M, Wagner M, Wilborn M, Bubic I, Jonjic S, Koszinowski U, Hengel H: A cytomegaloviral protein reveals a dual role for STAT2 in IFN-(gamma) signaling and antiviral responses. J Exp Med 2005; 201:1543-1553.

32 Petricoin EF 3rd, Ito S, Williams BL, Audet S, Stancato LF, Gamero A, Clouse K, Grimley P, Weiss A, Beeler J, Finbloom DS, Shores EW, Abraham R, Larner AC: Antiproliferative action of interferon-alpha requires components of T-cell-receptor signalling. Nature 1997; 390:629-632.

33 Sun S, Zhang X, Tough DF, Sprent J: Type I interferon-mediated stimulation of $\mathrm{T}$ cells by CpG DNA. J Exp Med 1998;188:2335-2342.

34 Buhler B, Keil GM, Weiland F, Koszinowski $\mathrm{UH}$ : Characterization of the murine cytomegalovirus early transcription unit $\mathrm{e} 1$ that is induced by immediate-early proteins. J Virol 1990;64:1907-1919.

35 Messerle M, Buhler B, Keil GM, Koszinowski $\mathrm{UH}$ : Structural organization, expression, and functional characterization of the murine cytomegalovirus immediate-early gene 3 . J Virol 1992;66:27-36.

36 Angulo A, Ghazal P, Messerle M: The major immediate-early gene ie 3 of mouse cytomegalovirus is essential for viral growth. J Virol 2000;74:11129-11136.

37 Hagemeier C, Walker S, Caswell R, Kouzarides T, Sinclair J: The human cytomegalovirus 80 -kilodalton but not the 72 -kilodalton immediate-early protein transactivates heterologous promoters in a TATA box-dependent mechanism and interacts directly with TFIID. J Virol 1992;66:4452-4456.

38 Caswell R, Hagemeier C, Chiou CJ, Hayward G, Kouzarides T, Sinclair J: The human cytomegalovirus $86 \mathrm{~K}$ immediate early (IE) 2 protein requires the basic region of the TATAbox binding protein (TBP) for binding, and interacts with TBP and transcription factor TFIIB via regions of IE2 required for transcriptional regulation. J Gen Virol 1993;74: 2691-2698.

39 Martinez FP, Cosme RS, Tang Q: Murine cytomegalovirus major immediate-early protein 3 interacts with cellular and viral proteins in viral DNA replication compartments and is important for early gene activation. J Gen Virol 2010;91:2664-2676.

40 Perez KJ, Martinez FP, Cosme-Cruz R, PerezCrespo NM, Tang Q: A short cis-acting motif in the M112-113 promoter region is essential for IE3 to activate M112-113 gene expression and is important for murine cytomegalovirus replication. J Virol 2013;87:2639-2647.

41 Lukac DM, Harel NY, Tanese N, Alwine JC: TAF-like functions of human cytomegalovirus immediate-early proteins. J Virol 1997;71: 7227-7239. 